\title{
Cypate and Cypate-Glucosamine as Near-Infrared Fluorescent Probes for In Vivo Tumor Imaging ${ }^{\mathrm{S}}$
}

\author{
Mona Doshi, Daniel A. Nierenberg, Orielyz Flores-Fernandez, Pragney Deme, \\ Edilu Becerra, Annette R. Khaled, and Sampath Parthasarathy
}

Burnett School of Biomedical Sciences, College of Medicine, University of Central Florida, Orlando, Florida

Received September 5, 2018; accepted February 25, 2019

\begin{abstract}
Near-infrared (NIR) imaging is a promising technique for use as a noninvasive and sensitive diagnostic tool. Although the NIR fluorescently labeled glucose analog glucosamine (cypateglucosamine) has applications in preclinical imaging, the transport pathways and fate of this probe in tissues remain unaddressed. Here, we have synthesized and characterized cypate and cypate-glucosamine conjugate (cy-2-glu), and investigated the probable transport pathways of these probes in vitro and in vivo. We compared uptake of the probes in the presence and absence of excess D-glucose, "saturated cypate" and palmitic acid in two normal-cancer cell line pairs: lung cancer (A549)-normal (MRC9) and prostate cancer (DU145)normal (BPH). Breast cancer (MDA-MB-231) and liver cancer (HepG2) cell lines were also examined. Results support use of

the glucose transport pathway by cy-2-glu and fatty acid transport pathway by cypate. Mass spectrometry data on the in vitro extracts revealed deamidation of cy-2-glu in prostate and liver cells, suggesting release of glucosamine. In vivo biodistribution studies in mice engrafted with breast tumors showed a distinct accumulation of cy-2-glu in liver and tumors, and to a lesser extent in kidneys and spleen. A negligible accumulation of cypate alone in tumors was observed. Analysis of urine extracts revealed renal excretion of the cy-2-glu probe in the form of free cypate, indicating deamidation of cy-2-glu in tissues. Thus, investigation of the metabolic pathways used by NIR probes such as cy-2-glu advances their use in the detection and monitoring of tumor progression in preclinical animal studies.
\end{abstract}

\section{Introduction}

Optical methods using near-infrared (NIR) probes, in the spectral range of $700-2500 \mathrm{~nm}$, are of interest due to features such as flexibility of use, cost-effectiveness, and minimal toxicity. Preclinical in vivo tumor imaging is a promising NIR probe application due to: 1) low autofluorescence from biomolecules (ensuring minimum background light), 2) low tissue absorbance (delivering high penetration of the light), 3) low light scattering (leading to high signal to noise ratio), and 4) nonradiation applications (limiting deleterious effects from radiation-based therapies). Noninvasive tumor detection by NIR probes has proven highly sensitive within various animal studies (Hilderbrand and Weissleder, 2010; Luo et al., 2011; Haque et al., 2017). Targeted and activated imaging of cancers was reported using NIR dyes (Escobedo et al., 2010; Hilderbrand and Weissleder, 2010; Owens et al., 2016). Conventional NIR imaging molecules include cyanine (Kim et al., 2005; Peng et al., 2005), phthalocyanine, porphyrine (Srinivasan et al., 2003; Tanaka et al., 2008; Xie et al., 2009), squaraine

https://doi.org/10.1124/mol.118.114199.

S This article has supplemental material available at molpharm. aspetjournals.org.
(Nakazumi et al., 2005; Volkova et al., 2007; Umezawa et al., 2008a), and BODIPY (boron-dipyrromethene) (Umezawa et al., 2008b, 2009; Donuru et al., 2010). Of these, the cyanine dye cypate has excellent optimal properties with a high extinction coefficient $\left[224,000(\mathrm{~mol} / \mathrm{L})^{-1} \mathrm{~cm}^{-1}\right]$ and two carboxylic groups suitable for conjugation with amine or hydroxyl groups.

Due to cypate's low tissue absorbance and thus minimal background it is well suited for in vivo imaging. This was first shown in studies conducted by Achilefu et al. $(2000,2005)$ in which peptide-conjugated cypate probes that targeted integrins, molecules involved in tumor-induced angiogenesis and metastasis, were tested for NIR imaging of A549 cells in nude mice. Subsequent studies have used glucosamine-conjugated cypate moieties for cancer-related imaging.

Ye et al. (2005) evaluated combinations of cypate-conjugated glucosamine moieties, observed their biodistribution in pancreatic cancer-bearing mice, and determined that the number of glucosamine moieties conjugated to cypate could influence cypate uptake in tumors in a similar manner. Cheng et al. (2006) studied Cy5.5-D-glucosamine (Cy5.5-2DG) and 2-( $N$-(7nitrobenz-2-oxa-1,3-diazol-4-yl)amino)-2-deoxyglucose (NBDG) probes, showing that NBDG, which has a smaller molecular weight compared with the Cy5.5-2DG, was more likely to use

ABBREVIATIONS: cy-1-glu, cypate-1-glucosamine; cy-2-glu, cypate-2-glucosamine; DCM, dichloromethane; DIEA, diisopropylethylamine; DMF, dimethylformamide; FDG, 2[F-18] fluoro-2-deoxy-D-glucose; FTIR, Fourier-transform infrared spectroscopy; GLUT, glucose transport protein; HBTU, $N, N, N^{\prime}, N^{\prime}$-tetramethyl-O-(1H-benzotriazol-1-yl)uronium hexafluorophosphate; HOBt, 1-hydroxybenzotriazole hydrate; LC-MS, liquid chromatography and mass spectrometry; NBDG, 2-(N-(7-nitrobenz-2-oxa-1,3-diazol-4-yl)amino)-2-deoxyglucose; NIR, near infrared; PA, palmitic acid; sat-cy, saturated cypate. 
glucose-based transport in U87MG human glioblastoma cells. Guo et al. (2012) examined the biodistribution and clear routes of an glucosamine-conjugated hydrophilic indocyanine green and glucosamine-conjugated lipophilic cypate probe in breast cancer and glioblastoma-bearing mice and found that indocyanine green-glucosamine probe cleared out faster than cypate-glucosamine probe. Korotcov et al. (2012) studied glucosamine-conjugated cypate biodistribution in prostate cancer-bearing mice, finding that cypate conjugated to two glucosamine moieties demonstrated higher tumor uptake and retention time than cypate conjugated to one glucosamine moiety.

As indicated by these studies, cypate and the cypate-conjugated probes have use in cancer imaging. Their optimum use depends on the biophysical properties of the probe (hydrophobicity, lipophilicity, molecular weight, number of targeting molecules, etc.). Additionally, knowledge of their transport pathways is equally important to make advancements in optimal probe design. One of the hallmarks of cancer is an altered metabolism leading to increased glucose uptake called the Warburg effect (Warburg, 1925; Hanahan and Weinberg, 2011; Liberti and Locasale, 2016). This phenomenon underlies malignant tissue detection by positron emission tomography (PET) scan using 2[F-18]fluoro-2-deoxy-D-glucose (FDG) (Som et al., 1980; Hoh et al., 1993; Lapela et al., 1995; Flamen et al., 2000; Phelps, 2000). Chemical exchange saturation transfer (CEST) magnetic resonance imaging also employs D-glucose, FDG, 2-deoxy-Dglucose, 3-O-methyl-D-glucose, glucosamine, and $\mathrm{N}$-acetyl glucosamine molecules (Zhang et al., 2003; Chan et al., 2011; Vinogradov et al., 2013; Walker-Samuel et al., 2013; Wu et al., 2016; Rivlin and Navon, 2018). Hence, use of these methods shows the effectiveness of targeting glucose metabolism for imaging probes, supporting our approach with cypate.

However, despite their value in imaging, the glucose- and carboxylate-based transport pathways of such imaging probes have yet to be investigated. To this end, we examined the biophysical characteristics of cypate and cypate-glucosamine (cy-2-glu) as well as their optical properties. We determined whether glucosamine-conjugated cypate transport into cells is glucose dependent or carboxylate dependent. We also characterized and analyzed the molecular changes in cypate and cy-2-glu in animals to determine the retention time and fate of the probes.

Understanding the transport mechanisms of cypate and cy-2-glu in malignant and non-malignant cells adds needed information on how the chemical structure of NIR probes affects their biodistribution. This information will advance the tissue-specific imaging and drug delivery application of NIR probes such as cy-2-glu, demonstrating that these conjugates have translational benefits.

\section{Materials and Methods}

\section{Materials}

We purchased 1,1,2-trimethyl benz[e]indole, 3-bromopropanoic acid, 1,2-dichlorobenzene, dichloromethane (DCM), acetonitrile, sodium acetate, glutaconaldehyde dianil hydrochloride, diisopropylethylamine (DIEA), acetic anhydride, dimethylformamide (DMF), $N, N$, $N^{\prime}, N^{\prime}$-tetramethyl- $O$-( $1 H$-benzotriazol-1-yl)uronium hexafluorophosphate (HBTU), 1-hydroxybenzotriazole hydrate (HOBt), glucosamine hydrochloride, DMSO, acetone, $\mathrm{Pd} / \mathrm{C}(10 \% \mathrm{Pd})$, and liquid chromatography and mass spectrometry (LC-MS) solvents from Sigma Aldrich
(St. Louis, MO). Hydrochloric acid was purchased from Fisher Scientific (Pittsburgh, PA). Diethyl ether was purchased from VWR (Radnor, PA). Ethanol was purchased from Pharmaco-Aaper (Brookfield, CT).

\section{Methods}

\section{Synthesis of Cypate, Cy-2-glu, and Saturated Cypate.}

Synthesis of 1,1,2-trimethyl benz[e]indole-3-propanoic acid. As previously described elsewhere, we synthesized 1,1,2-trimethyl benz[e] indole-3-propanoic acid (Ye et al., 2005). Briefly, $4 \mathrm{~g}$ of 1,1,2-trimethyl benz[e]indole ( $19.11 \mathrm{mmol})$ were dissolved in $20 \mathrm{ml}$ of 1,2-dichlorobenzene in a conical flask to obtain a dark yellow solution. Four grams of 3-bromopropanoic acid ( $26.15 \mathrm{mmol})$ were added to this solution. The solution then was heated to $110^{\circ} \mathrm{C}$ with continuous stirring for 18 hours before cooling to room temperature, at which time the solution turned dark gray-green. This solution was vacuum filtered through Whatman filter paper. The white colored precipitate was washed with DCM by trituration and transferred to a glass petri dish. This synthesis yielded $5.5 \mathrm{~g}$ of product.

Synthesis of cypate. Cypate was synthesized as previously described elsewhere (Ye et al., 2005). Briefly, $4.1 \mathrm{~g}$ of 1,1,2-trimethyl benz[e] indole-3-propanoic acid (synthesized as described in the previous section) was added to a $50-\mathrm{ml}$ acetonitrile-water $(47.5 \mathrm{ml}$ of acetonitrile $+2.5 \mathrm{ml}$ of deionized water) solvent system in a round-bottom boiling flask. We added $1.6 \mathrm{~g}$ of sodium acetate to this solution and refluxed at $170^{\circ} \mathrm{C}$. In a conical flask, we dissolved $1.42 \mathrm{~g}(4.98 \mathrm{mmol})$ of glutaconaldehyde dianil hydrochloride in $10 \mathrm{ml}$ of DCM.

While this solution was kept on ice, $1.3 \mathrm{~g}(10 \mathrm{mmol})$ of DIEA was added to it. In a separate glass vial, $0.6 \mathrm{~g}$ of acetic anhydride was mixed in $2.5 \mathrm{ml}$ of DCM. This solution was then added dropwise to the solution containing glutaconaldehyde dianil hydrochloride and stirred at room temperature at a speed of $100 \mathrm{rpm}$ for 1 hour.

After mixing, the resultant mixture was added dropwise to the refluxing solution listed above. The reflux was continued for 16 hours at $170^{\circ} \mathrm{C}$. The dark-green precipitate that formed was filtered through a glass funnel with Whatman filter paper. It was washed with acetonitrile, $5 \%$ hydrochloric acid, and finally with ether. The product was then transferred into a glass petri dish and air-dried for 2 days. The clumps were separated with a spatula to obtain a dark-green, fine cypate powder.

Synthesis of cy-2-glu. Cypate was conjugated to glucosamine as previously described elsewhere (Ye et al., 2005). Briefly, $30 \mathrm{mg}$ of cypate $(0.048 \mathrm{mmol})$ were dissolved in $5 \mathrm{ml}$ of DMF in a round-bottom flask. We dissolved $41 \mathrm{mg}$ of HBTU in $1 \mathrm{ml}$ of DMF, which was added to the cypate solution. We dissolved $14.8 \mathrm{mg}$ of $\mathrm{HOBt}(0.096 \mathrm{mmol})$ in $1 \mathrm{ml} \mathrm{DMF}$ and added this to the cypate solution. The final volume of this solution was brought to $10 \mathrm{ml}$ by adding DMF. This solution was then chilled to $-5^{\circ} \mathrm{C}$.

In a glass vial, $48 \mathrm{mg}$ of glucosamine $(0.266 \mathrm{mmol})$ were dissolved in $2 \mathrm{ml} \mathrm{DMSO}$, and $76 \mu \mathrm{l}$ of DIEA $(0.436 \mathrm{mmol})$ were added to it. The glucosamine solution was added to the cold cypate solution in one portion and stirred at room temperature for 2 hours. The reaction mixture was added to $50 \mathrm{ml}$ of ether in a conical flask under vigorous stirring. Upon product precipitation, the excess ether was decanted.

To remove DMSO completely from the product, acetone was added to the precipitate; after brief shaking, the acetone was removed to prevent product dissolution. The product was placed in a glass petri dish and air-dried for 1 day to yield $23.8 \mathrm{mg}$ of crystalline cy-2-glu.

Synthesis of nonfluorescent, saturated cypate. For subsequent cell culture experiments involving the uptake of the NIR fluorescent probe cypate, a nonfluorescent, saturated cypate (sat-cy) was synthesized to act as a competitive inhibitor. To synthesize sat-cy, we dissolved $1 \mathrm{mg}$ of cypate in $2 \mathrm{ml}$ ethanol in a 5 -ml round-bottom flask and added approximately $1 \mathrm{mg}$ of $\mathrm{Pd} / \mathrm{C}(10 \% \mathrm{Pd})$. In a glass cylinder, we mixed zinc granules with $50 \mathrm{ml}$ of $6 \mathrm{~N}$ hydrochloric acid. Once hydrogen gas had formed, it was passed through the reaction mixture overnight while stirring at room temperature. The reaction mixture was then filtered and centrifuged to remove $\mathrm{Pd} / \mathrm{C}$. Ethanol was evaporated completely to yield sat-cy. 
Cell Culture and Experiments.

Cell culture. A549 (human lung cancer), MRC9 (human lung normal), BPH (human prostate normal), MDA-MB-231 and luciferase-expressing MDA-MB-231-luc2 (human breast cancer), and HepG2 (human liver cancer) cell lines were cultured in DMEM with $10 \%$ FBS in a humidified atmosphere of $95 \%$ air $/ 5 \% \mathrm{CO}_{2}$ at $37^{\circ} \mathrm{C}$. DU145 (human prostate cancer) cells were grown in RPMI medium supplemented with $10 \% \mathrm{FBS}$ in a humidified atmosphere of $95 \%$ air $/ 5 \% \mathrm{CO}_{2}$ at $37^{\circ} \mathrm{C}$. THLE-2 (normal human liver) cells were cultured in Airway Epithelial Cell Basal Medium (American Type Culture Collection [ATCC], Manassas, VA) supplemented with a bronchial epithelial cell growth kit (ATCC) and grown under a humidified atmosphere. Cell lines were purchased from ATCC and frozen after three passages. All cell lines were used from frozen stocks for no more than 9-12 passages.

In vitro cypate and cy-2-glu uptake assays. We plated $1 \times 10^{6}$ of each cell type on individual $35-\mathrm{mm}$ glass-bottom petri dishes. After 24 hours, the cell lines were incubated for 2 hours with a $10 \mu \mathrm{M}$ solution of cypate/cy-2-glu in phenol red free medium. After treatment, the cells were either washed $3 \times$ with PBS and fixed with $4 \%$ paraformaldehyde for imaging or had their intracellular cypate and cy-2-glu contents collected for spectroscopy and/or LC-MS analysis.

For intracellular cypate and cy-2-glu collection, the treated cells were harvested with $0.25 \%$ trypsin, centrifuged, and washed with PBS. The pellet was resuspended in $1 \mathrm{ml}$ of methanol/acetonitrile/ water solvent system $(2: 2: 1, \mathrm{v} / \mathrm{v})$ and vortexed for 30 seconds. The cells were then freeze-fractured by chilling the samples in liquid nitrogen for 1 minute, allowing them to return to room temperature and then sonicated them for 10 minutes. The described procedure was repeated 3 times to ensure maximum extraction of the dyes.

Afterward, the samples were placed in $-20^{\circ} \mathrm{C}$ for 1 hour to precipitate the cellular matrix. The samples were centrifuged for 15 minutes at $13,000 \mathrm{rpm}$ at $4^{\circ} \mathrm{C}$. The supernatant was collected and evaporated to dryness under gentle stream of nitrogen gas $\left(\mathrm{N}_{2}\right)$. The residues were dissolved in $100 \mu \mathrm{l}$ of acetonitrile/water $(1: 1, \mathrm{v} / \mathrm{v})$ to remove any remaining cell debris. The samples were centrifuged at $13,000 \mathrm{rpm}$ for 15 minutes at $4^{\circ} \mathrm{C}$. The supernatant was separated and dried completely under $\mathrm{N}_{2}$. The dried samples were resuspended in $400 \mu \mathrm{l}$ of methanol and stored until further analysis.

Competitive uptake of cypate and cy-2-glu with excess D-glucose, sat-cy, and $P A$. To assess which transport mechanisms may be involved in the cellular uptake of cypate and cy-2-glu, uptake assays as described in In Vitro Cypate and cy-2-glu Uptake Assays were performed in the presence of excess D-glucose, sat-cy, or PA. For each experiment, cells were cultured in DMEM alone or supplemented with $5 \mathrm{mM}$ D-glucose, $100 \mu \mathrm{M}$ sat-cy, or $100 \mu \mathrm{M}$ PA in phenol red-free medium for 2 hours. After treatment, the cells were fixed for imaging or had their intracellular cypate and cy-2-glu contents collected for analysis via spectroscopy and/or LC-MS.

\section{Animal Experiments.}

In vivo D-glucose competition for cy-2-glu uptake. To access how Dglucose competes against cy-2-glu uptake in vivo, tumors were implanted into mice. Briefly, $100 \mu \mathrm{l}$ of $8 \times 10^{5}$ luciferase-expressing breast cancer cells (MDA-MB-231 Luc2) in Matrigel (Corning Life Sciences, Tewksbury, MA) were implanted orthotopically in the mammary fat pad of JAX (Jackson Laboratory, Bar Harbor, ME) Foxn1nu/Foxn1nu nude 2.5-month-old female mice using a 26.5G BD $1 \mathrm{ml}$ insulin U-100 syringe.

As a pilot study, two nude mice with breast tumors of comparable size $\left(\sim 250 \mathrm{~mm}^{3}\right)$ were selected. In one of the mice, $100 \mu \mathrm{l}$ of $20 \mathrm{mM}$ D-glucose in PBS was injected intravenously. After 15 minutes, $10 \mathrm{nmol}$ of cy-2-glu in $100 \mu \mathrm{l}$ PBS was administered in both mice through tail vein injection. After 20 minutes, $15 \mathrm{mg} / \mathrm{ml}$ of luciferin in PBS $(100 \mu \mathrm{l})$ was injected in the intraperitoneal cavity. Imaging was performed in a Bruker Xtreme in vivo imaging system with Bruker MI SE software (Bruker, Billerica, MA).

The mice were anesthetized using $2 \%$ isoflurane. Animals were then imaged as described below. Because the fluorescence intensity from liver was very high, it was impeding imaging of other organs or tissues. The liver area was covered with a black strip, and the exposure time for fluorescence was increased to 2 minutes to collect maximum signal from rest of the organs. The parameters for bioluminescence and reflectance images remained unchanged. Overlaying of the images was performed with the same software.

Biodistribution of cy-2-glu and cypate in organs. We implanted $8 \times$ $10^{5}$ luciferase-expressing breast cancer cells (MDA-MB-231 Luc2) orthotopically in the mammary fat pad of JAX (Jackson Laboratory) Foxn1nu/Foxn1nu nude 2.5-month-old female mice. We intravenously injected $10 \mathrm{nmol}$ cy-2-glu/cypate in $100 \mu \mathrm{l}$ PBS in these mice. Experiments were performed at 1 hour after injection and then every 24 hours for 6 days. For each time point, three mice were used with similar-sized tumors $\left(\sim 250 \mathrm{~mm}^{3}\right)$. Fifteen minutes before imaging, urine was collected from the mice and kept in the refrigerator until further analysis. We injected $15 \mathrm{mg} / \mathrm{ml}$ luciferin in PBS $(100 \mu \mathrm{l})$ into the intraperitoneal cavity 10 minutes before in vivo imaging. The mice were anesthetized with $2 \%$ isoflurane and imaged as described below. A black strip was used again to block the liver as described previously.

After in vivo imaging, mice were sacrificed by cervical dislocation, and their organs were isolated and imaged. After ex vivo imaging, cy-2-glu and cypate were extracted from the organs and urine by cutting the organs into small pieces in glass petri dishes with small scissors and transferring them into homogenizing tubes. We added $1 \mathrm{ml}$ of deionized water to the tubes, and the samples were homogenized for 5 minutes. After this, $3 \mathrm{ml}$ of methanol were added to each tube, and the samples were homogenized again for 5 minutes. These solutions were centrifuged for 10 minutes at $3000 \mathrm{rpm}$. The supernatants were collected and stored at $4^{\circ} \mathrm{C}$ overnight to allow precipitate formation. The solutions were centrifuged, and the supernatants were collected and dried with $\mathrm{N}_{2}$ gas. We added $400 \mu \mathrm{l}$ of methanol to dried samples and centrifuged again. These collected supernatants contained only cy-2-glu or cypate.

For urine extraction, $500 \mu \mathrm{l}$ of urine were collected in a glass tube. We next added $1 \mathrm{ml}$ of deionized water and $2 \mathrm{ml}$ of methanol. After precipitate formation, the samples were centrifuged, and the supernatants were collected. The supernatants were dried with $\mathrm{N}_{2}$ gas, and $400 \mu \mathrm{l}$ of methanol were added. Solution spectroscopy and mass spectrometry were performed on the organ and urine extraction samples. All animal work was conducted under the approval of the Institutional Animal Care and Use Committee (IACUC) of the University of Central Florida.

\section{Chemical Characterization.}

Spectroscopy. Absorption was analyzed via UV-visible spectroscopy performed in methanol in a 1-cm path-length submicro quartz cuvette from Spectrecology (Wesley Chapel, FL) with a 6850 Jenway spectrophotometer (Cole-Parmer, Staffordshire, United Kingdom) using Prism software (GraphPad Software, San Diego, CA). The fluorescence spectroscopy was performed in methanol in a 1-cm pathlength submicro quartz cuvette with PTI QM-400 fluorimeter (Horiba Canada, Burlington, ON) using FelixGX 4.3.6904 software (Horiba Canada). The detectors used to collect the fluorescence from the samples were 1) a multimode photomultiplier tube detector for UV-visible range $(135-700 \mathrm{~nm})$ and 2) a TE cooled InGaAs detector for the NIR range $(700-1700 \mathrm{~nm})$. The acquisition time was 0.5 seconds at a $1-\mathrm{nm}$ interval. Fourier-transform infrared spectroscopy (FTIR) was used to analyze the spectra of the cypate and cy-2-glu samples and was recorded with a Perkin Elmer Spectrum 100 (PerkinElmer, Waltham, MA).

Liquid chromatography and mass spectrometry. An Agilent 1200 series high-performance liquid chromatography system consisting of a G1379B degasser, a G1311A quaternary pump, an HTC PAL autosampler, and a G1316A column compartment coupled to a 6520 quadrupole time of flight (QTOF) mass spectrometer (Agilent, Santa Clara, CA) was used to characterize and analyze the samples. Compounds were separated on Zorbax Eclipse Plus C18 (150 mm L× $4.6 \mathrm{~mm}$ ID, $5 \mu \mathrm{m}$ PS) column using a binary mobile phase gradient program to elute the components: pump A (acetonitrile) and pump B (water), both containing $0.1 \% \mathrm{HCOOH}$. The gradient program was as follows: $90 \%$ B: $0-5$ minutes; $10 \%$ B: $5-12$ minutes; $10 \% \mathrm{~B}, 12-$ 18 minutes; $90 \%$ B: $18-18.1 ; 10 \%$ B: $18.1-23$ minutes. At the end of 
each run, the column was washed for 3 minutes with a solvent composition consisting of 50\% isopropyl alcohol, 30\% methanol, $20 \%$ water, and $0.1 \% \mathrm{HCOOH}(\mathrm{v} / \mathrm{v})$. The column was operated at $40^{\circ} \mathrm{C}$ with a constant mobile phase flow rate of $750 \mu \mathrm{l} / \mathrm{min}$.

The mass spectrometer was operated in electrospray ionization positive mode over a mass range of $50-1700 \mathrm{~m} / \mathrm{z}$. The mass spectrometer was tuned and calibrated at $4 \mathrm{GHz}$ high-resolution mode at low mass $50-1700 \mathrm{~m} / \mathrm{z}$ range with a manufacturer calibration solution (G1969-85000; Agilent). The reference mass solution was continuously infused through the second nebulizer to ensure better mass accuracy throughout the analysis. The electrospray ionization source parameters were optimized and operated under the following conditions. Capillary voltage was $3.5 \mathrm{kV}$; nitrogen was used as a drying and nebulizer gas, and the values were set at $13 \mathrm{l} / \mathrm{min}$ and $55 \mathrm{psi}$, respectively; the source temperature was set at $320^{\circ} \mathrm{C}$; and the fragmentor and skimmer voltages were set at 100 and $65 \mathrm{~V}$, respectively. The MS data were collected and processed using MassHunter qualitative analysis software, version B.07.00 (Agilent).

Imaging.

Confocal microscopy. Confocal microscopy was performed on in vitro cell culture experiments, as described earlier. A Zeiss AXIO Observer.Z1 microscope with a Zeiss LSM 710 laser system was used for imaging (Carl Zeiss Microscopy GmbH, Jena, Germany). The images were acquired with Zeiss ZEN 2010.B SP1 software. The objective lenses used were Plan-Apochromat 20X/0.8 M27 and PlanApochromat 63X/1.40 oil DIC M27. For Z-stack image acquisition of A549 cells, we acquired 15 confocal slices at $0.64 \mu \mathrm{m}$ intervals. Cypate and cy-2-glu were excited with $647 \mathrm{~nm}$ wavelength; sat-cy and PA were excited with $488 \mathrm{~nm}$ wavelength.

In vivo and ex vivo imaging of whole animals and individual organs. In vivo imaging on mice was performed using a Bruker Xtreme in vivo imaging system with Bruker MI SE software. Images were collected in three channels. 1) For the fluorescence channel, the excitation wavelength was $760 \mathrm{~nm}$, and the emission was collected using a 830-nm long-pass filter with exposure time of 2 minutes. 2) For the bioluminescence channel, the bioluminescence from luciferin in the tumor was collected using a 2-minute exposure time. 3 ) For the reflectance channel, the gray reflectance image was collected with a 5 -second exposure time.

Because the fluorescence intensity from liver was very high, it was impeding the imaging of other organs or tissues. The liver area was covered with a black strip, and the exposure time for fluorescence was increased to 2 minutes to collect maximum signal from the rest of the organs. The parameters for bioluminescence and reflectance images remained unchanged. Overlaying of the images was done in the same software. These three images were overlaid and analyzed for colocalization of cy-2-glu/cypate in the tumors or tissues.

After in vivo imaging, the mice were sacrificed by cervical dislocation, and their organs were isolated and imaged. Organ images were collected in two channels. 1) For the fluorescence channel, the excitation wavelength used was $760 \mathrm{~nm}$, and the emission was collected with $830 \mathrm{~nm}$ long-pass filter using an exposure time of 2 minutes. 2) For the reflectance channel, the gray reflectance image was collected with a 5 -second exposure time.

Statistical Analysis. The in vitro experiments were run in triplicate with a minimum of three repeats. The in vivo experiments consisted of $n=$ 3 animals per experimental group. Statistical analyses were performed using unpaired Student's $t$ test in GraphPad (GraphPad Software). $P<$ 0.05 was considered statistically significant.

Data availability. The raw/processed data required to reproduce these findings cannot be shared at this time as the data also forms part of an ongoing study.

\section{Results}

\section{Synthesis and Characterization of Cypate and cy-2-glu}

The NIR dye cypate and the cypate-glucosamine conjugates were synthesized and recovered as pure products by modifying the procedure reported by Ye et al. (2005) as described in the Materials and Methods section. Cypate-glucosamine contained either one carboxylic acid conjugated to one glucosamine (cy-1glu) or two carboxylic acids each conjugated to one glucosamine moiety (cy-2-glu) (Scheme 1). The molar ratios for synthesis of cy-1-glu yielded a mixture of cy-1-glu, cy-2-glu, and unreacted cypate (Supplemental Fig. 1A) whereas the molar ratios for synthesis of cy-2-glu resulted in only one product, cy-2-glu (Supplemental Fig. 1B). For this reason, cy-2-glu was used for subsequent studies.

We evaluated the optical properties of cypate and cy-2-glu by absorption and fluorescence spectroscopy (Supplemental Fig. 2, A and B). Cypate and cy-2-glu absorbance spectra have $\lambda_{\max }$ at $784 \mathrm{~nm}$ and a shoulder at $722 \mathrm{~nm}$ (Supplemental Fig. 2A). The fluorescence profile of cypate and cy-2-glu starts at $800 \mathrm{~nm}$ and tails off at $1000 \mathrm{~nm}$, with two emission maxima at $831 \mathrm{~nm}$ and at $935 \mathrm{~nm}$, depicting a wide NIR spectral window for imaging and diagnostic purposes (Supplemental Fig. 2B).

The normalized absorption and fluorescence spectra revealed that conjugation of glucosamine to cypate had no adverse effect on the spectral properties of cypate. Neither the spectral profile nor the maxima of absorbance and fluorescence changed due to the conjugation of glucosamine to cypate, although a slight quenching in cypate was noted (data not shown).

We checked the formation of an amide bond between the carboxylic group of cypate and the amine group of glucosamine by recording the FTIR spectrum of cy-2-glu and comparing it with the FTIR spectrum of cypate. A medium band for secondary N-H stretch at $3282 \mathrm{~cm}^{-1}$ showed the presence of secondary amide between the carboxylic group of cypate and the amine group of glucosamine (Supplemental Fig. 2C). This band can be seen only in the spectrum of cy-2-glu and is absent in cypate. The expanded spectrum in the region from 2000 to $700 \mathrm{~cm}^{-1}$ revealed $\mathrm{C}=\mathrm{O}$ stretches and amide bends (Supplemental Fig. 2D). The appearance of $\mathrm{C}=\mathrm{O}$ amide stretch at $1651 \mathrm{~cm}^{-1}$ in cy-2-glu along with the absence of $\mathrm{C}=\mathrm{O}$ carboxylic acid stretch, which is seen in cypate spectrum at $1718 \mathrm{~cm}^{-1}$, confirmed amide bond formation between cypate and glucosamine. The appearance of N-H bend I $\left(1618 \mathrm{~cm}^{-1}\right)$ and II $\left(1538 \mathrm{~cm}^{-1}\right)$ in cy-2-glu supported this conclusion.

We also performed proton and carbon NMR for structure determination (Supplemental Fig. 3, A and B). Once cypate and cy-2-glu probes were synthesized, purified, and characterized, we next evaluated the transport pathway of these probes and assessed their biodistribution and elimination in vivo.

\section{Competitive Inhibition of Uptake of cy-2-glu in Cancer Cell Lines Indicates Glucose Transport Pathway Involvement}

As both glucose and glucosamine are taken up in the cells using glucose transport proteins (Calvo et al., 2010), we determined whether the presence of excess of D-glucose would competitively inhibit the uptake of cy-2-glu. This competitive uptake inhibition was observed in the A549, HepG2, DU145, and MDA-MB-231 cell lines (Fig. 1). The fluorescence intensities obtained from the confocal images were used as a direct measurement for in vitro cellular uptake of cy-2-glu. Decreases in fluorescence intensities for cy-2-glu in the presence of excess (5 mM supplemented) D-glucose as compared 


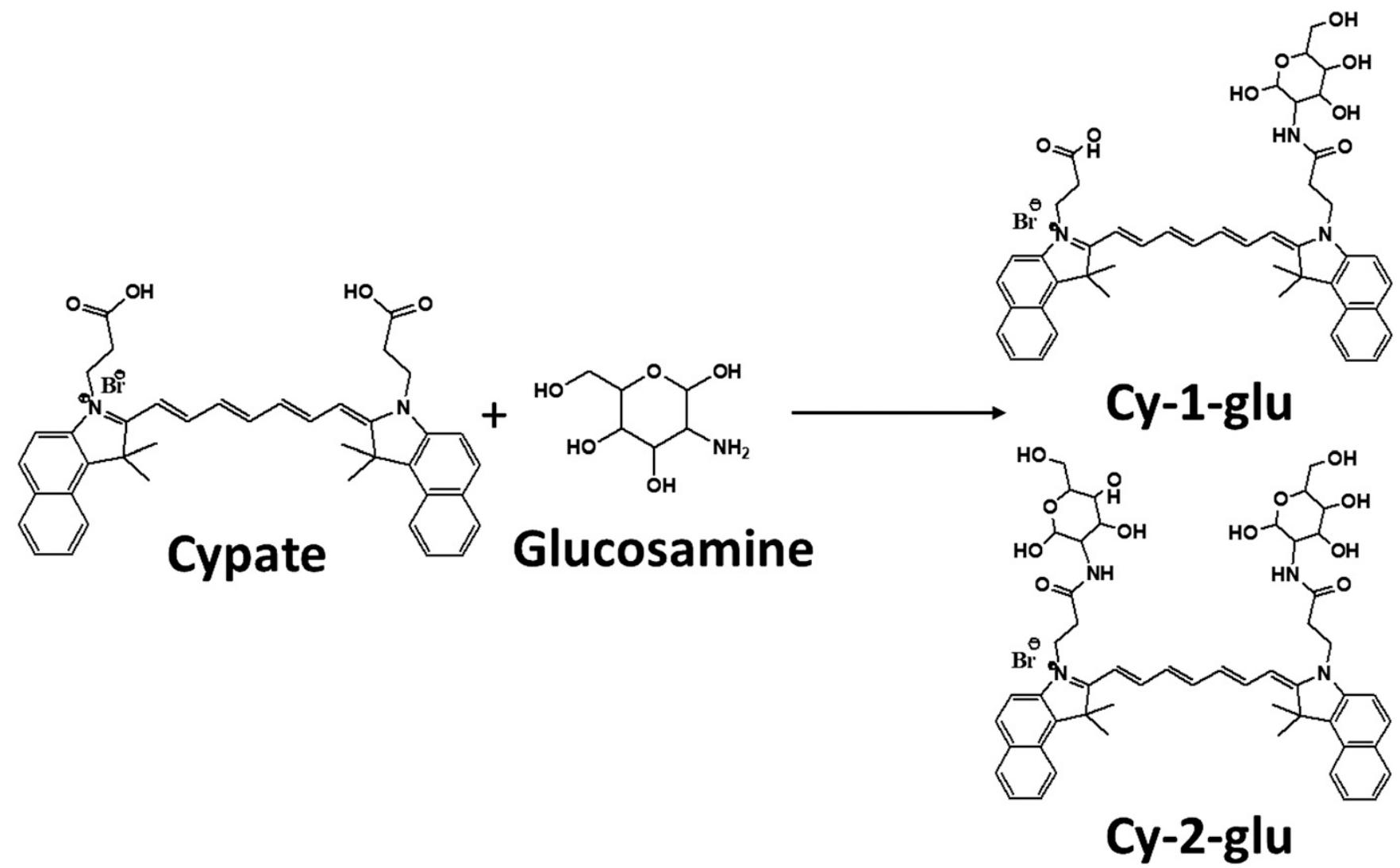

Scheme 1. Chemical structures of cypate, glucosamine, cy-1-glu, and cy-2-glu.

with absence of D-glucose (no D-glucose supplementation) (Fig. 1A) served as evidence of competitive inhibition. Cypate alone was not inhibited in the presence of excess D-glucose (Fig. 1B). Z-stack analysis of A549 cells further showed that the probes did not adhere to the plasma membrane but rather were distributed throughout the interior of cells (Supplemental Fig. 4).

Solution spectroscopy of cellular extracts determined the quantitative uptake of cypate and cy-2-glu. The absorption values at $784 \mathrm{~nm}$ and the emission intensities at $831 \mathrm{~nm}$ for cy-2-glu (Fig. 1, C and D) and cypate (Fig. 1, E and F) in the presence or absence of excess D-glucose are plotted in bar graphs. As with the confocal images (Fig. 1, A and B), the spectroscopy data showed a decrease in absorbance and fluorescence of cy-2-glu in cancer cell lines when excess D-glucose was present in the medium. Data from cypate in the presence of excess D-glucose showed negligible change in absorbance and fluorescence. These results suggest that cy-2-glu uses a transport mechanism involving glucose whereas cypate uses a non-glucose-utilizing pathway for transport.

The fluorescence intensities of cy-2-glu and cypate in the nontransformed cell lines MRC9 and BPH showed no visible change in the microscopy data in presence or absence of excess D-glucose. However, the fluorescence spectroscopy data for $\mathrm{BPH}$ did reveal a decrease in uptake of cypate in presence of excess D-glucose (Fig. 1E), suggesting that these immortalized cells could be more metabolically active. This is likely the result of higher anaerobic metabolic activity in cancerous tissue in response to the demands of growth under low oxygen conditions (Warburg, 1925, 1956; Hanahan and Weinberg, 2011; Liberti and Locasale, 2016).

Though cypate lacks sugar moieties, we did observe cypate uptake by cancer cells. One explanation for this could be interactions with cypate carboxylates on the cell surface of cancer cells. The reduced negative charge on the surface of cancer cells as compared with normal or nontransformed cells could lessen the repulsion for carboxylate groups, leading to a higher uptake of cypate. Note however that these findings may only be relevant for cells grown under in vitro culture conditions.

We compared the quantitative data of all the cancer and nontransformed cell extracts (Supplemental Fig. 5). The absorbance spectra for cypate and cy-2-glu extracted from these cell lines are shown in Supplemental Fig. 5, A and B. with the absorbance and fluorescence maxima of the spectra plotted as a bar graph (Supplemental Fig. 5, C and D). These data confirmed the increased uptake of cypate and cy-2-glu in cancer cells in comparison with nontransformed cells.

\section{Competitive Uptake Inhibition of Cypate Indicates Involvement of Fatty Acid Transport Pathway}

To determine whether cypate uses carboxylate transporters for transport and uptake, we performed a competitive uptake inhibition experiment using: 1) palmitic acid (PA), a fatty acid and 2) saturated cypate (sat-cy), a non-NIR fluorescent analog of cypate.

Inhibition with Fatty Acid PA. If the carboxylic acid groups on cypate were used for transport purposes, the 
A

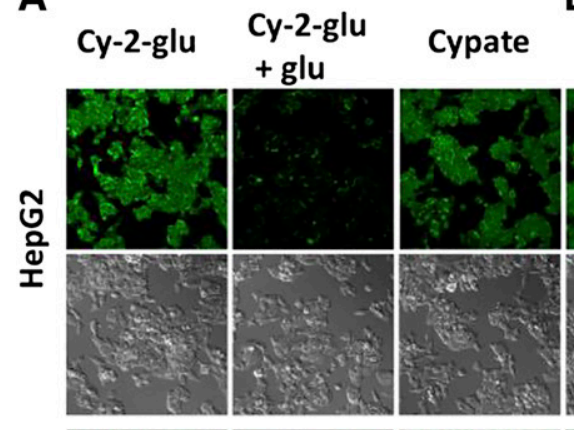

B
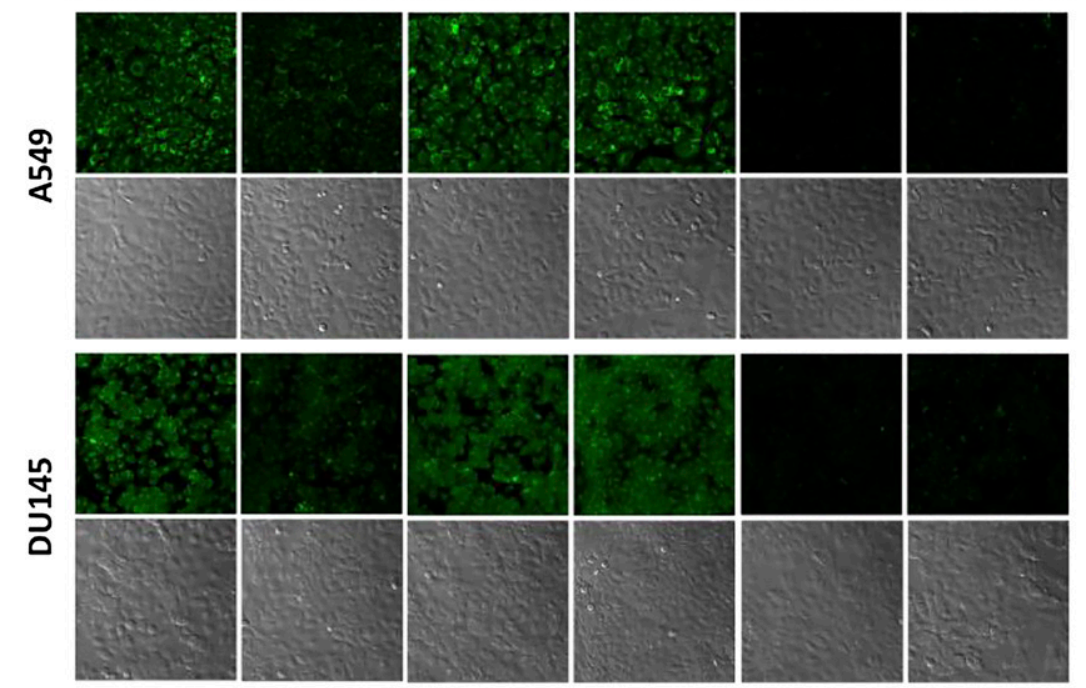

商
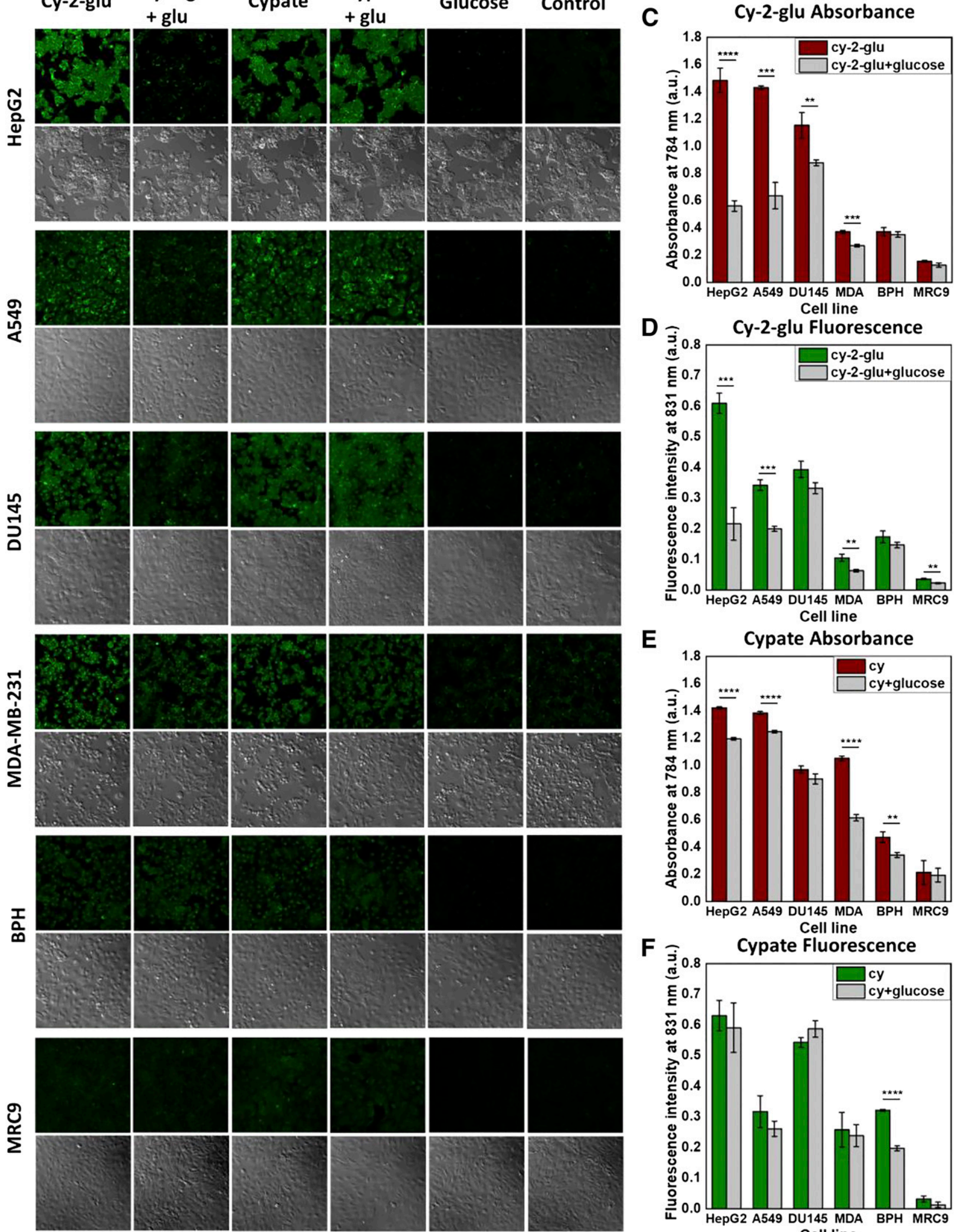

\section{$\mathbf{F}$}

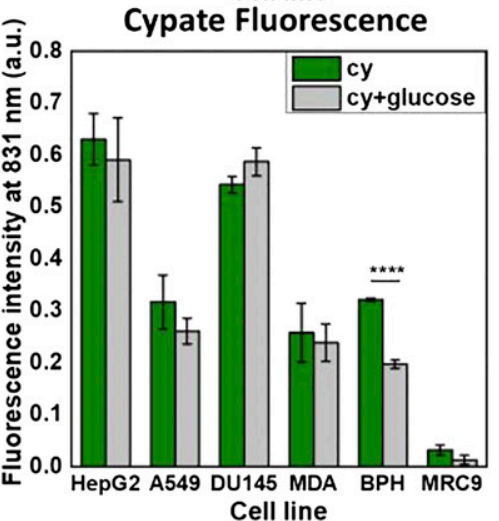

Fig. 1. Presence of excess D-glucose competes with cy-2-glu for uptake in cell lines. Confocal fluorescence and corresponding diffraction interference contrast images of the cell lines incubated with cy-2-glu (A) or cypate (B) in the presence or absence of excess D-glucose. Green color represents the fluorescence from cy-2-glu or cypate. Solution spectroscopy data on cell extracts for cy-2-glu (C and D) or cypate (E and F). (C) The cy-2-glu absorbance values in the absence (red) or presence (gray) of D-glucose. (D) The cy-2-glu fluorescence values in absence (green) or presence of D-glucose (gray). (E and F) Cypate absorbance or fluorescence, respectively, in the same manner as graphs in $C$ and $D$. $* * P<0.01 ; * * * P<0.001$; ****P<0.0001. Error bars represent S.D. $(n=3)$. 
presence of excess of fatty acid $(100 \mu \mathrm{M})$, which uses carboxylates for fatty acid transport, would competitively inhibit the uptake of cypate. To test this, we incubated A549 cells with cypate in the presence of $100 \mu \mathrm{M}$ PA. Confocal fluorescence images showed reduced fluorescence intensity when PA was present in the medium (Fig. 2B). Conversely, when the carboxylic acid groups of cypate were modified (amide bond in cy-2-glu), the presence of PA did not result in competitive inhibition of cypate uptake (Fig. 2A), indicating that carboxylic acid groups are integral to cypate for transport and cellular uptake.

Inhibition with Saturated Cypate as a Non-NIR Fluorescent Analog of Cypate. To further validate that cypate is taken up via fatty acid transporters, we used sat-cy as a non-NIR fluorescent analog of the NIR fluorescent cypate (Fig. 3), exploiting the optical properties of cypate. Cypate owes its NIR optical properties to the $\pi$-conjugated system present within the molecule. Hence, we disrupted the $\pi$-conjugation by reducing the double bonds in the glutaconaldehyde dianil moiety, which acts as the bridge between the two indole molecules. Hydrogenation of two double bonds added four hydrogen atoms to the molecule, breaking the $\pi$-conjugation system and blue shifting the spectra of cypate. We called this new molecule saturated cypate (sat-cy). The synthesis of sat-cy is depicted in Fig. 3A. The addition of four hydrogen atoms to the cypate molecule was confirmed by mass spectrometry (Fig. 3, B and C). The molecular ion at $625 \mathrm{~m} / \mathrm{z}$ for cypate (Fig. 3B) shifted to a molecular ion at $629 \mathrm{~m} / z$ for sat-cy (Fig. 3C). Thus, sat-cy has no absorbance or emission in the NIR region (700-1000 nm) (Fig. 3, D and E). Incubation of A549 cells with excess sat-cy resulted in competitive inhibition of cypate uptake, similar to addition of excess PA (Fig. 2, A and B, cy-2-glu + sat-cy and cypate + sat-cy) confirming the use of carboxylic acid groups by cypate for transport and cellular uptake.

The absorbance values and fluorescence intensities obtained via UV-visible and fluorescence spectroscopy of cy-2-glu (Fig. 2, $\mathrm{C}$ and D) and cypate (Fig. 2, E and F) from A549 cell extracts correlated with the data collected from confocal microscopy analysis.

\section{Deamidation of cy-2-glu in Prostate and Liver Cell Lines}

After determining that cy-2-glu and cypate molecules use different pathways for transport, we next asked if the cypate probes underwent any molecular change once inside of cells. We characterized extracts from cells treated with cypate probes by mass spectrometry and observed that the mass spectra of extracts from DU145 and BPH incubated with cy-2glu showed the presence of cypate $(625 \mathrm{~m} / z)$ along with cy-2glu $(947 \mathrm{~m} / z)$, suggesting hydrolysis of cy-2-glu in these cells (Fig. 4A). Conversely, extracts from these cell lines incubated with cypate only showed the presence of the ion at $625 \mathrm{~m} / z$ that is related to cypate, suggesting that no molecular changes in cypate occurred (Fig. 4B).

In another experiment, instead of analyzing the cell extracts, we analyzed the metabolites released into the cell culture medium. For this, we incubated the liver cell line THLE-2 with cy-2-glu for 2 hours before the addition of fresh medium. These cells were incubated for another 24 hours. Metabolites released into the culture medium were then analyzed via LC-MS. We observed the peak for cypate, indicating release of the dye from cells after cy-2glu was converted into cypate by hydrolysis (Fig. 4D).

We also performed this experiment with the MDA-MB-231 breast cancer cell line (data not shown), but no deamidation was observed. The results show that cy-2-glu is hydrolyzed in the liver but not in the tumors, which has implications for the in vivo use of this probe.

\section{In Vivo Monitoring of cy-2-glu}

We investigated the accumulation and retention of cypate and cy-2-glu in nude mice orthotopically implanted with MDA-MB231-luc2 breast cancer cells. Upon detection of tumors, we intravenously injected the mice with $10 \mathrm{nmol}$ of cy-2-glu or cypate, and the fluorescence intensity of the probes was monitored in vivo for 6 days. Mice with cy-2-glu were evaluated 1 hour after the injection, then every 24 hours for 6 days (Fig. 5A).

Because the accumulation of cypate alone in the tumor was negligible, we monitored these mice only 24 hours after the injection (Fig. 5B). Not unexpectedly, the accumulation of probes in the liver produced a high degree of fluorescence that impeded imaging the fluorescent signal from tumors and other organs (data not shown). To remedy this, the liver area was covered with a black strip to image the rest of the animal.

The overlaying tumor bioluminescence (red) with cy-2-glu/ cypate fluorescence (green) resulted in a yellow image (i.e., colocalization) that visualized the accumulation of both signals and indicated the presence of cy-2-glu within tumors. Maximum fluorescence intensity of cy-2-glu in tumors was seen at 24 hours after injection, which reduced gradually and almost vanished from the tumor by day 6 (Fig. 5A). A trace amount of fluorescent signal was still detectable from the liver after day 6 .

The in vivo accumulation of cy-2-glu but not cypate within the tumor contrasted with the in vitro observation where cypate was equally taken up by the cancer cells. This discrepancy may be due to variances in tissue culture conditions compared with the tumor microenvironment (e.g., nutrient availability).

\section{Ex Vivo Imaging and Extraction of Probes for Evaluation of Biodistribution of cy-2-glu and Cypate in Various Organs}

To evaluate the biodistribution of cy-2-glu and cypate in the organs, we performed ex vivo imaging of tumors and organs. A subset of mice were euthanized at each time point and had their organs harvested and imaged (Fig. 5, C and D). The fluorescence intensities are shown in a gradient manner; red represents the highest intensity, and blue represents the lowest intensity.

The liver had the highest accumulation of cy-2-glu and cypate at all time points. Over the 6 days observed, the fluorescence intensity from cy-2-glu gradually decreased in all the organs (Fig. 5C) and almost vanished except within the liver which retained trace fluorescence. The absorbance data of the reconstituted solutions of organ extracts are shown in Fig. $5 \mathrm{E}$ and are directly correlated with in vivo and ex vivo image analysis.

The concentrations of cy-2-glu in the organs were calculated from the absorbance values of the organ extracts. Only the liver, tumor, kidneys, and spleen retained detectable cy-2-glu concentrations. Of note, the tumor was the second highest tissue for cy-2-glu accumulation. The absorbance values of cy-2glu from all other organs were below the limit of detection.

\section{Use of Excess of D-Glucose to Reduce the Uptake of cy-2-glu in Liver}

To reduce the uptake of cy-2-glu by the liver and improve tumor imaging, we performed a competitive inhibition experiment with $100 \mu \mathrm{l}$ of $20 \mathrm{mM}$ D-glucose in PBS via intravenous 

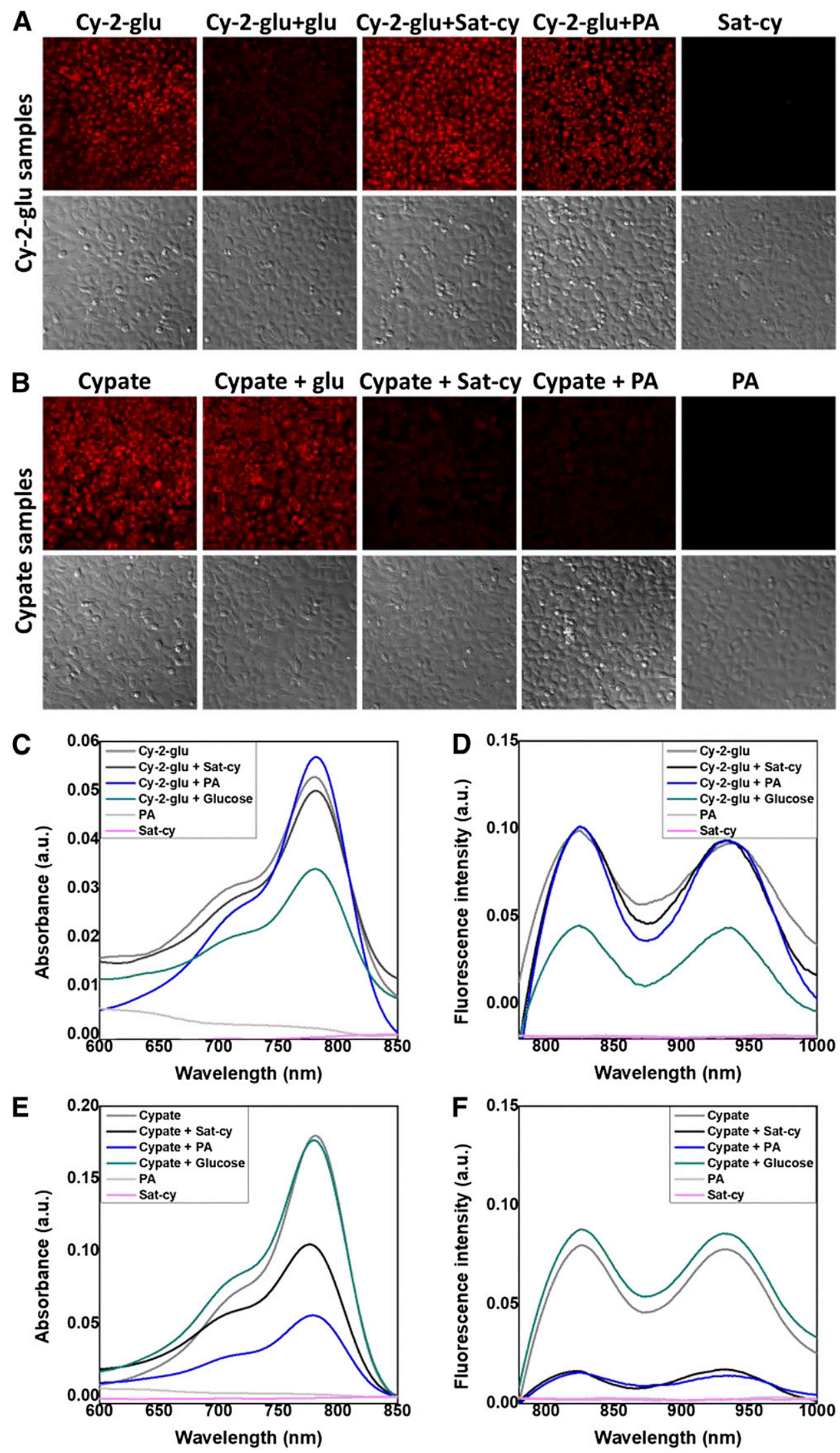

Fig. 2. Sat-cy and PA compete for internalization with cypate. Confocal fluorescence and corresponding diffraction interference contrast images of A549 cell line with cy-2glu (A) and cypate (B) in the presence or absence of excess D-glucose (glu), sat-cy, and PA. (C) The cy-2-glu absorbance spectra. (D) The cy-2-glu fluorescence spectra. (E and F) The cypate absorbance and fluorescence spectra, represented in the same manner as $C$ and $D$, respectively. Cy-2-glu and cypate were extracted from the A549 cell lines $(n=3)$. 
A

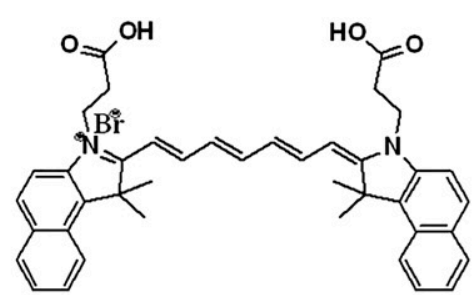

Cypate

B

$x 10^{6}$

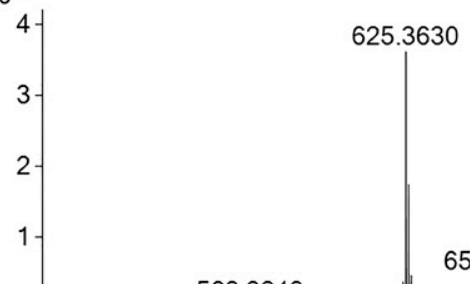

$\mathrm{Pd} / \mathrm{C}(10 \% \mathrm{Pd})$

Ethanol

$\mathrm{H}_{2}$

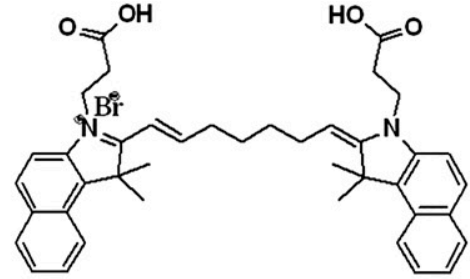

Saturated cypate

520540560580600620640660680700720740760780800820840860880900920940960980 Counts vs. Mass-to-Charge $(\mathrm{m} / \mathrm{z})$

C $\times 10^{4}$

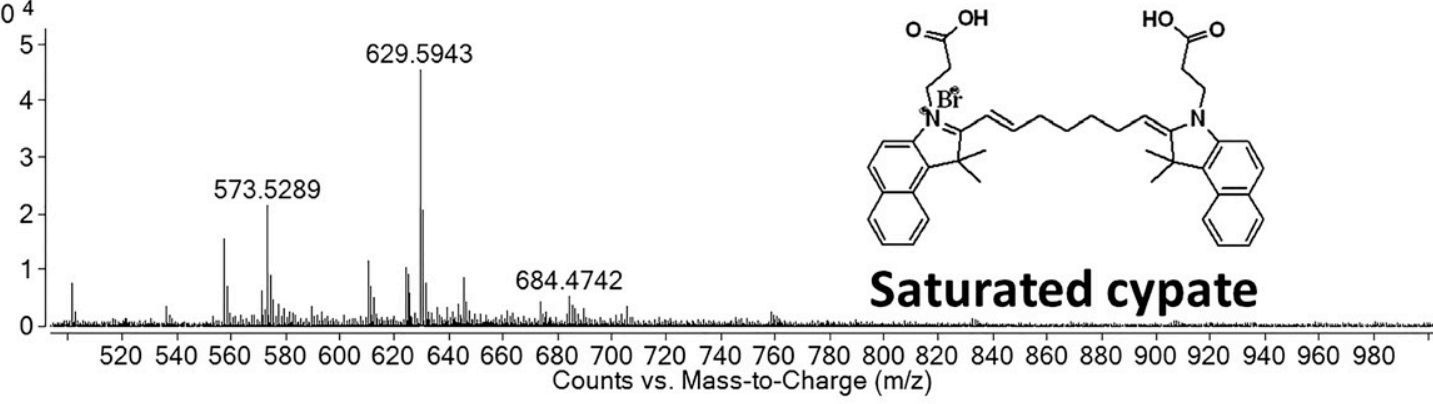

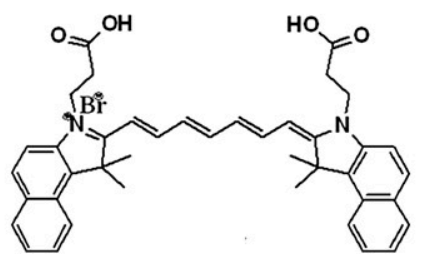

Cypate
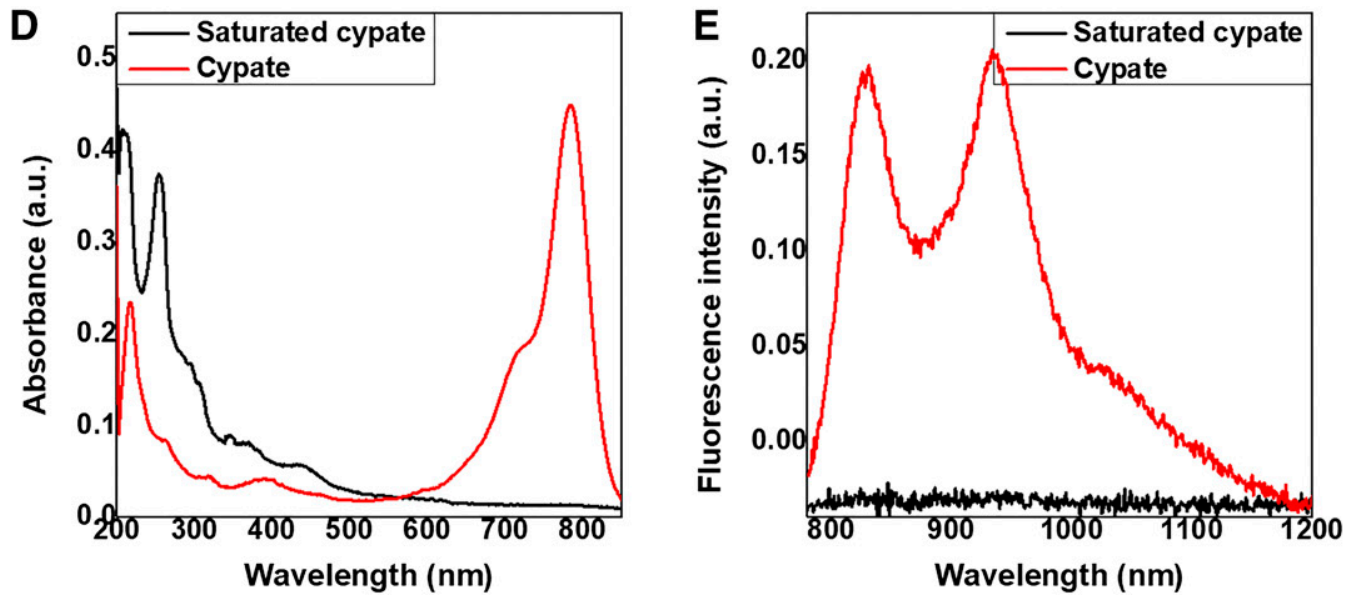

Fig. 3. Synthesis and characterization of sat-cy. Reduction of cypate with $\mathrm{Pd} / \mathrm{C}$ (10\% Pd) in ethanol and passing hydrogen gas through the solution to form sat-cy (A). Mass spectra of cypate at $\mathrm{m} / z 625$ (B) and at $\mathrm{m} / z 629$ for sat-cy (C). Absorbance spectra (D) and fluorescence spectra (E) of cypate (red line) and sat-cy (black line).

supplementation in nude mice. We anticipated that saturation of the liver with D-glucose before administration of cy-2-glu could reduce the uptake of cy-2-glu by the liver and decrease the fluorescence intensity from this organ.

After D-glucose administration, the presence of cy-2-glu in the liver was visibly reduced at the 20-minute and 4-hour time points (Fig. 6A). However, the fluorescence intensity emanating from the liver was still too high and obscured the rest of the organs. Thus, the liver area was covered with a black strip, and the exposure time was increased from 10 seconds to 2 minutes to collect ample signal from the mammary tumor and other tissues.

As seen from the images at 20 minutes and 4 hours, fluorescence intensity was reduced when excess D-glucose was injected (Fig. 6B). At 24 hours the effect of D-glucose before administration wore off, and the cy-2-glu accumulation in both mice was nearly equivalent. These results support our previous in vitro findings that cy-2-glu was capable of using a 


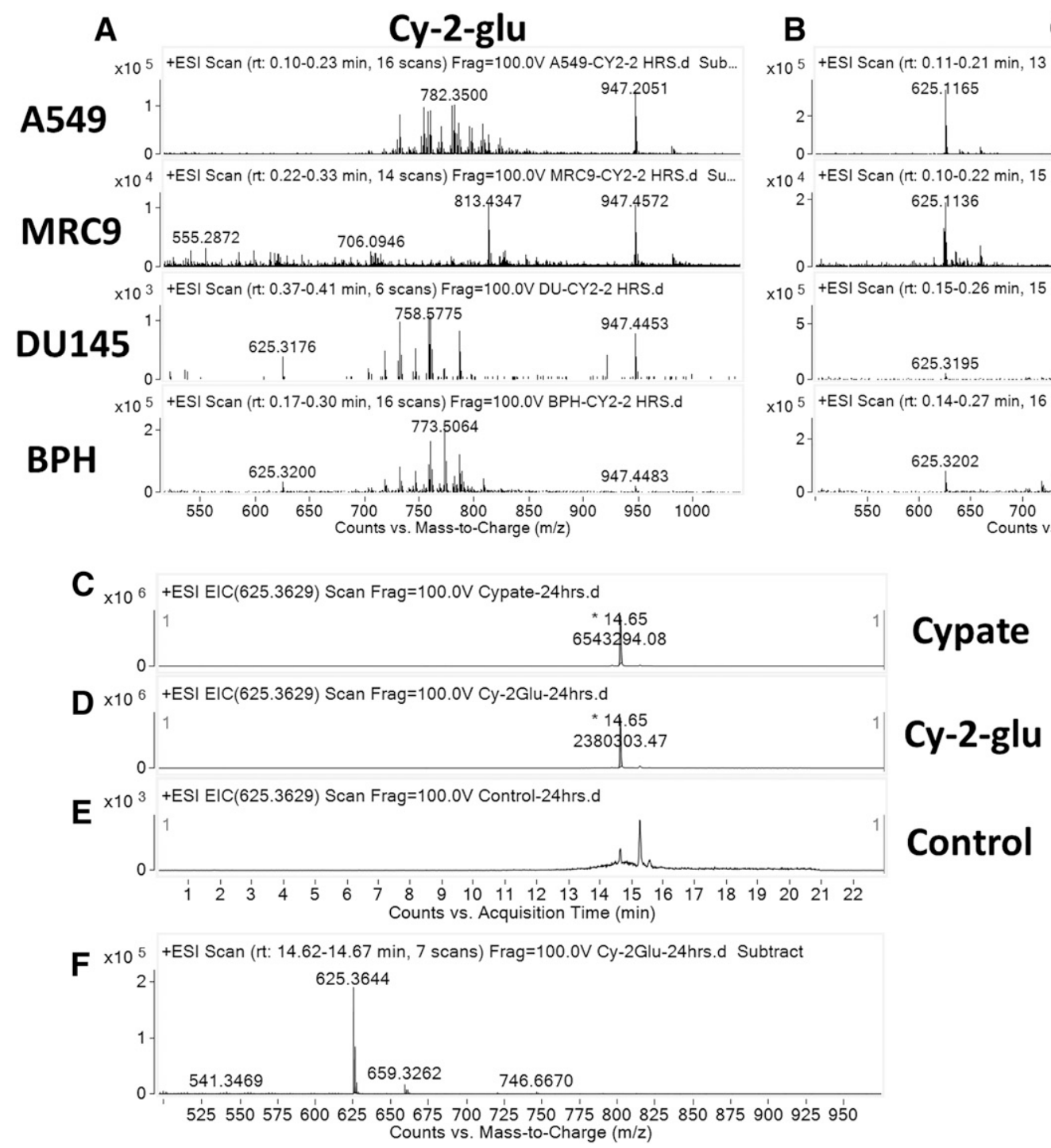

Fig. 4. Hydrolysis of cy-2-glu observed in prostate and liver cell lines. Mass spectra of dyes extracted from cancer and normal cell lines. (A) Extractions from cell lines incubated with cy-2-glu. A549 and MRC9 show the ion at $m / z$ 947 corresponding to cy-2-glu, while DU145 and BPH show the ions at $m / z$ 947 and 625 corresponding to cy-2-glu and cypate, respectively. (B) Extractions from cell lines incubated with cypate. The ion at $m / z 625$ corresponding to cypate is observed in all the cell lines. (C-E) The representative LC-HRMS extracted ion chromatograms of cypate at $m / z 625.3629$ ([M-H] ion). Liver cell line THLE-2 incubated with (C) cypate, (D) cy-2-glu, (C) control and medium evaluated for presence of cypate. (F) Corresponding mass spectra of extracted ion chromatograms.

glucose-based transport mechanism for entering the cells and suggest that this approach could be optimized in the future to improve cy-2-glu imaging.

\section{Metabolic Fate of the Probes}

To determine the retention time of cy-2-glu in vivo and discern the in vivo fate of the probe in mice, we analyzed urine extracts from mice treated with cy-2-glu. The absorbance data on the urine samples over a 6-day period indicated that the probe was continuously excreted from the body (Fig. 7A). Mass spectrometry data on specimens from mice 24 hours after injection of cy-2-glu revealed a peak at $625 \mathrm{~m} / z$ (Fig. 7B), suggesting deamidation of cy-2-glu to cypate occurred during the excretion process. This supports our previous in vitro findings of cy-2-glu deamidation in liver cells (Fig. 4D).

\section{Discussion}

Our study of the transport pathway and biodistribution of cy-2-glu and cypate demonstrates how imaging probes can be made to take advantage of cancer metabolism. Glucose is transported into cells through glucose transport proteins (GLUTs). Malignancies promote blood vessel formation to increase oxygen and nutrient uptake. Consequently, malignant tissues may alter their metabolism and produce lactic acid through aerobic glycolysis. This lactic acid is responsible for the membrane translocation of GLUTs, which in turn increases glucose uptake within the cells (Medina and Owen, 2002). Thus, increased energy utilization promotes proliferation and leads to enhanced glucose uptake and overexpression of glucose transporters. Due to ligand promiscuity, mannose, galactose, and glucosamine can also be transported via GLUTs (Calvo et al., 2010). 
A

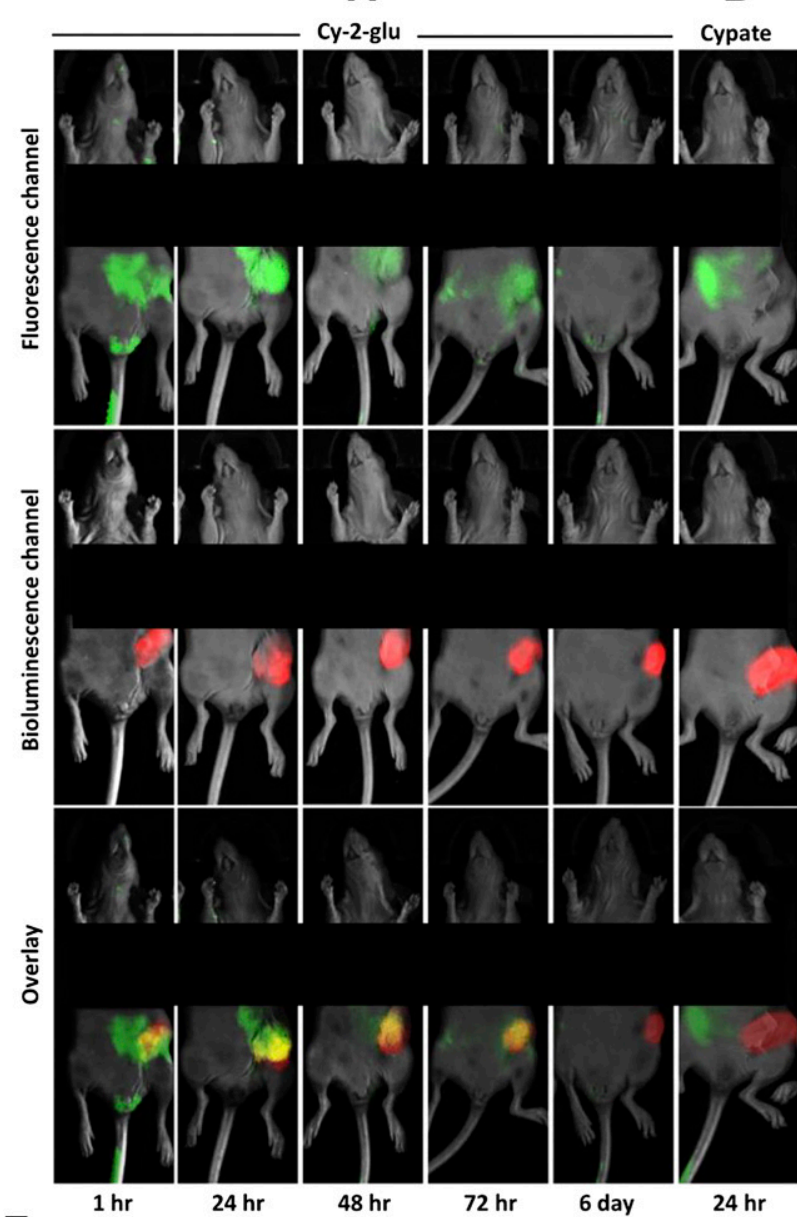

E

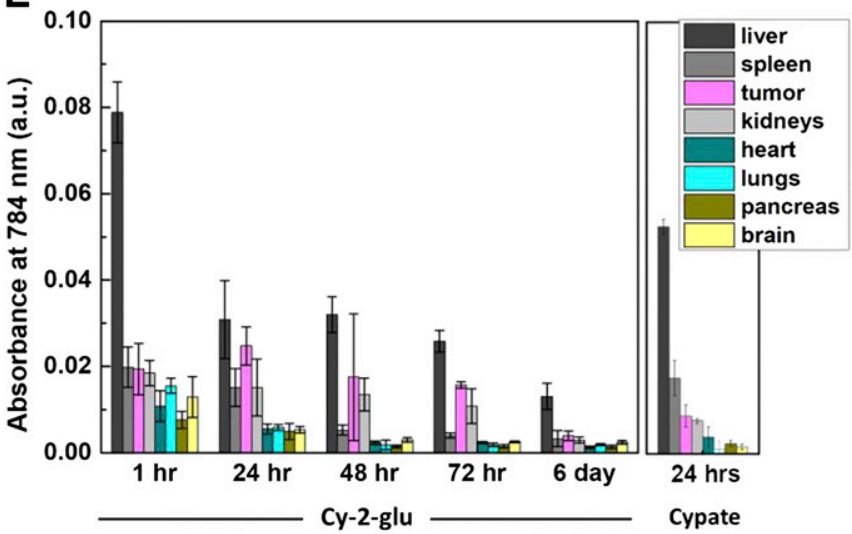

C

Fluorescence images Reflectance images
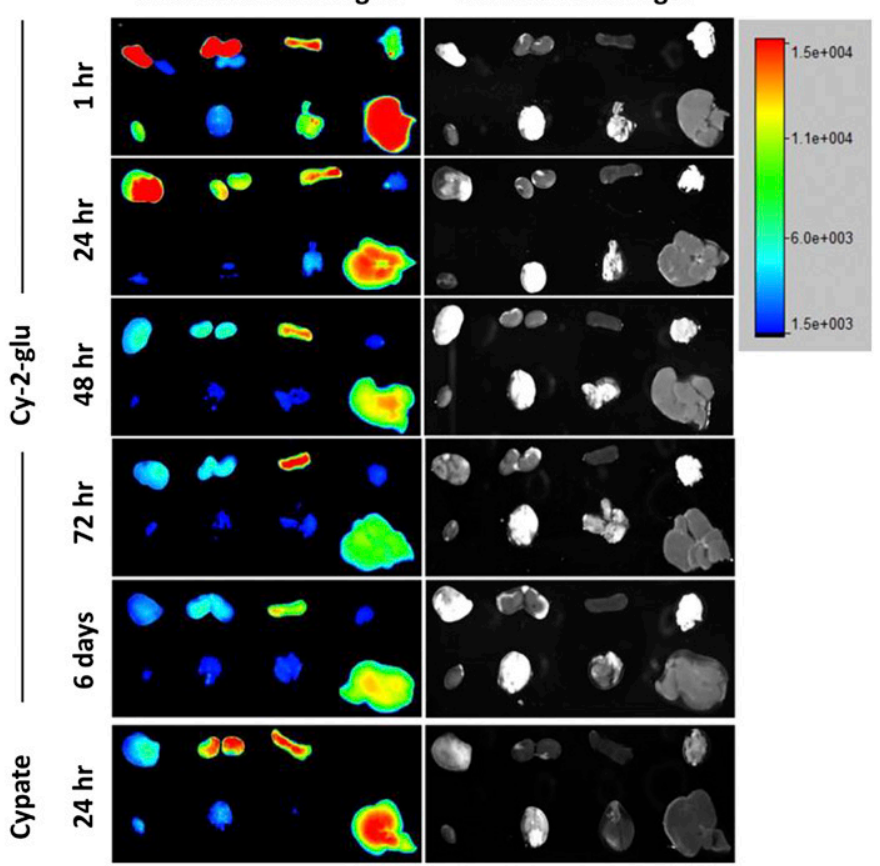

g

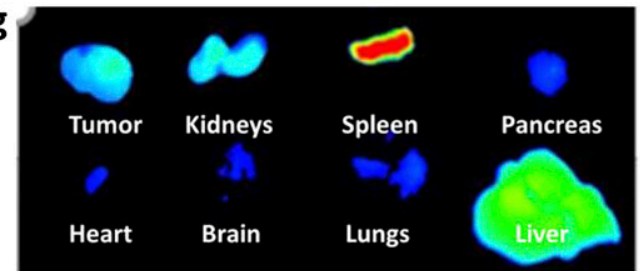

$\mathbf{F}$

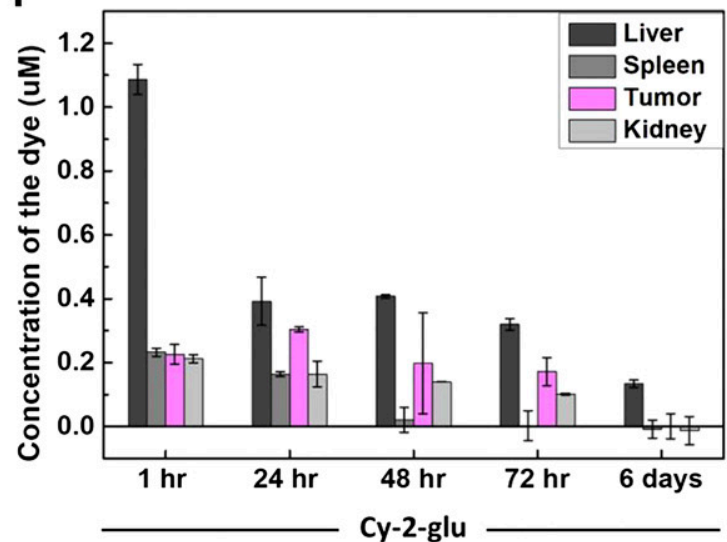

Fig. 5. Retention and biodistribution of cy-2-glu and cypate over 6 days. (A and B) In vivo images of nude mice bearing MDA-MB-231-luc2 tumors administered with (A) cy-2-glu and (B) cypate. The liver area is covered with a black strip to block the strong fluorescence signal. Imaging for cy-2-glu injection was performed at 1 hour after injection, followed by 24-hour periods for 6 days, and for cypate at 24 hours after injection. Green color indicates cy-2-glu/cypate fluorescence, red color indicates bioluminescence from tumor due to luciferase expression, and gray color represents the reflectance image. An overlay of these images yields colocalization of cy-2-glu/cypate in tumors (yellow color). (C and D) Ex vivo imaging. Fluorescence (C) and corresponding reflectance (D) images of organs isolated from mice at each time point (excitation wavelength $760 \mathrm{~nm}$, emission $830 \mathrm{~nm}$ long pass). Red indicates the highest and blue indicates the lowest fluorescence intensity. The organs are placed in the order shown $(\mathrm{G})$. ( $\mathrm{E}$ and F) Biodistribution in organs. Absorbance values of extracted probes plotted in bar graphs for each time point (E). Concentrations of cy-2-glu in liver, spleen, tumor, and kidney (F), as calculated from the absorbance values obtained from $E$ and from the calibration curve of cy-2-glu concentrations (Supplemental Fig. 6). Error bars represent S.D. ( $n=3$ mice per group).

In our study, we correlated cy-2-glu uptake in cancer cell lines with the GLUT overexpression on their surfaces. For example, A549 cells, which are reported to overexpress GLUT4 and GLUT1, have enhanced cy-2-glu uptake as compared with their normal counterpart, which does not express GLUT4 (Ong et al., 2008; O'Byrne et al., 2011). In prostate cells, GLUT1 expression increases with advanced malignancies (Effert et al., 2004). Based on the previously 
A

\section{B}
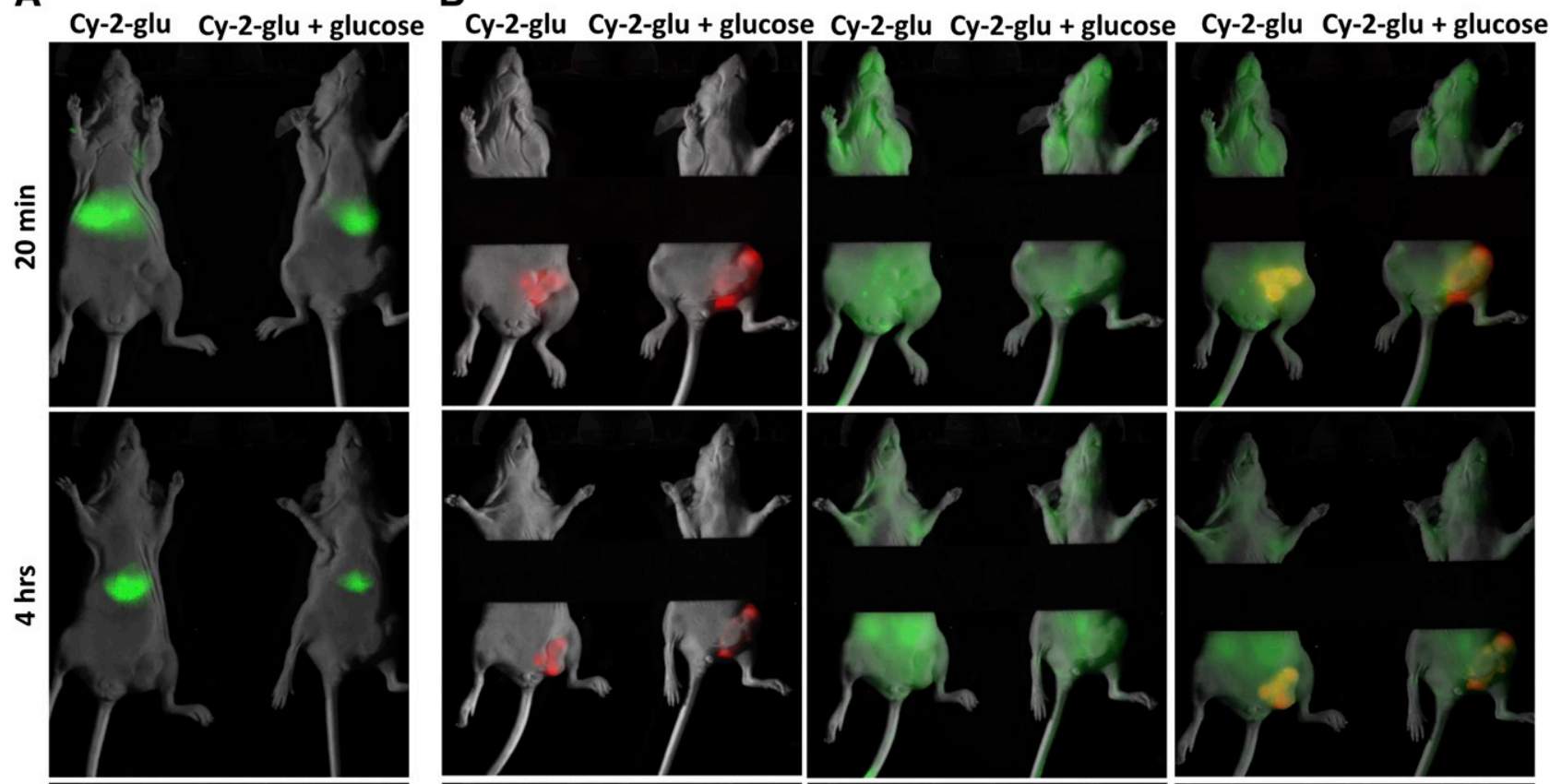

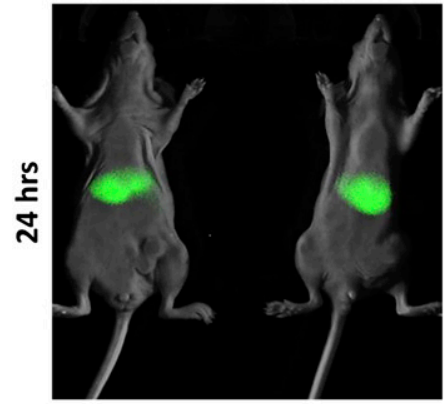

Fluorescence channel

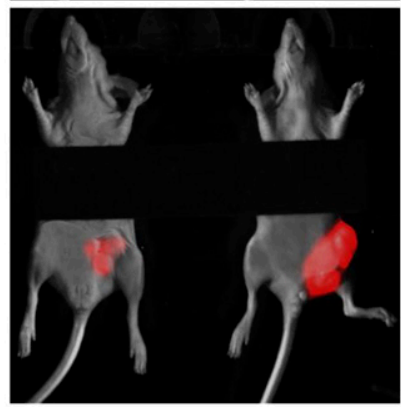

Bioluminescence channel

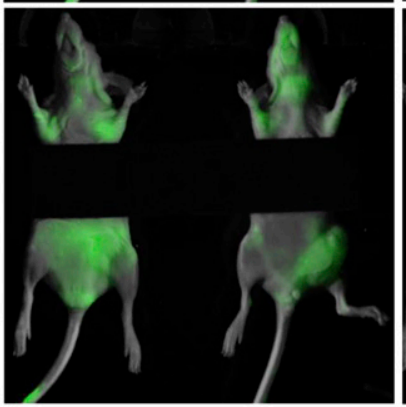

Fluorescence channel

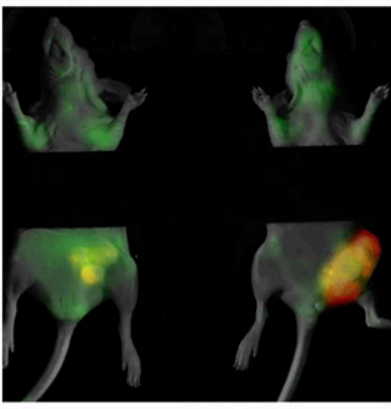

Overlay

Fig. 6. In vivo competitive effect of glucose on cy-2-glu uptake. Images of mice at 20 minutes, 4 hours, and 24 hours after injection of cy-2-glu. Green color represents fluorescence of cy-2-glu, red color represents bioluminescence from the tumor, and gray is the reflectance image. Here, (cy-2-glu + glucose) indicates injection of excess D-glucose 15 minutes before administration of cy-2-glu. (A) Overlay of fluorescence and reflectance images showing cy-2-glu in the liver (exposure time: 10 seconds). (B) Overlay of fluorescence, bioluminescence, and reflectance images showing colocalization of cy-2-glu and the tumor in yellow color. In $B$ the liver is covered with a black strip (exposure time: 2 minutes). ( $n=2$ mice/group)

published studies and the results obtained by our study, we can infer that the higher glucose requirement of cancer cells could increase cy-2-glu uptake in malignant cells as compared with their normal, nontransformed counterparts.

Others have reported that the addition of glucose can competitively inhibit glucosamine transport (Ebstensen and Plagemann, 1972; Plagemann and Erbe, 1973). We performed a similar competition experiment to demonstrate that cy-2-glu employs a glucose-utilizing transport pathway. Using D-glucose, the uptake of cy-2-glu was inhibited in cancer cells. In contrast, using a different cancer cell line, prostate cancer PC3-luc cells, Korotcov et al. (2012) reported that cypate-glucosamine probe uptake was not affected by D-glucose. These different outcomes could result from cellspecific effects or the in vitro cell culture conditions used. Hence, cancer cells may possess distinct surface charges that affect macromolecule uptake.

Another aspect that could impact the uptake of dyes like cypate was presented by Cheng et al. (2006), who suggested, using U87MG gliolblastoma cells, that a dye's molecular weight could affect its transport pathway. Interestingly, in our cancer cells, we did not observe any change in glucosamine transport due to attachment of the large cypate molecule; in the noncancer cell lines, the NIR signal was too low to detect any difference in cy-2-glu uptake in presence and absence of $\mathrm{D}$-glucose. The in vivo uptake of cy-2-glu in mice also displayed competitive inhibition by D-glucose during the first 4 hours of the administration of cy-2-glu. Cy-2-glu accumulation showed a considerable reduction in the liver and also in the tumor.

Our intent in performing this experiment was to determine whether the liver would take up more preinjected D-glucose from the blood than the tumor, competitively inhibiting cy-2-glu uptake and reducing the intensity of the very bright liver fluorescent signal. Although this proved to be the case, the fluorescent intensity of the cy-2-glu signal in the tumor was also reduced. However, Korotcov et al. (2012) had performed similar in vivo studies and showed that no competitive inhibition of cypate-glucosamine occurred at 24 hours after injection with D-glucose, and we also observed that at the 24-hour time point the inhibition effect of D-glucose wore off. It is possible that this approach to reducing liver fluorescence and improving detection of the tumor signal could work for 


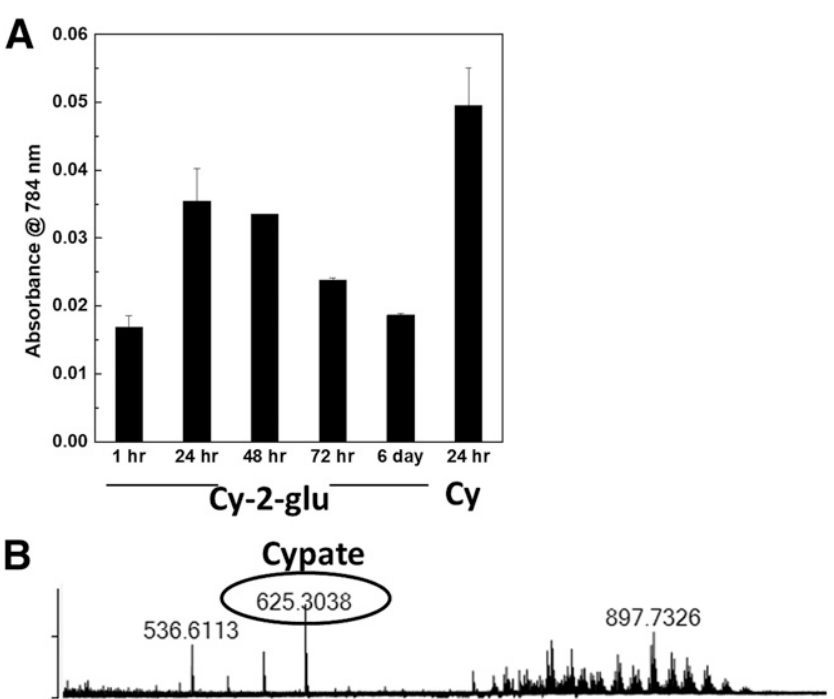

Fig. 7. Characterization of the urine extracts. (A) Absorbance values at $784 \mathrm{~nm}$ for cy-2-glu/cypate extracted from urine at increasing time points. (B) Mass spectrometry data on urine extracts of mice injected with cy-2-glu at the 24-hour time point showing molecular ion peak at $625 \mathrm{~m} / \mathrm{z}$ for cypate. Error bars represent S.D. ( $n=3$ mice per group).

other cancer cell lines with different metabolic profiles, which can be evaluated in future studies. The presented in vitro and in vivo results confirm the use of GLUT-based pathway for cy-2-glu transport and uptake by cancer cells.

One reason for the difference in the in vitro uptake of cypate in cancer cells compared with normal cells could be the interaction of negatively charged cypate with the cell membrane. Cancer cells have less negative charge on the surface despite the surface area being similar (James et al., 1956; Purdomet al., 1958; Cook and Jacobson, 1968; Zhang et al., 2008), which leads to reduced repulsion between cypate and the cancer cell surface, enhancing uptake. In our in vitro studies we observed that cypate and cy-2-glu were similarly taken up by the cancer cells, but in vivo we found that the cypate accumulation in the tumor was less than the cy-2-glu accumulation. This was also reported by Korotcov et al. (2012), who showed that amount of cypate taken up by cancer cells was less than the cypate-glucosamine conjugates. The reduced uptake of cypate in the tumor in vivo, compared with cy-2-glu, could be explained by the difference in the chemical structure of these two cypate forms. The protein makeup associated with cypate and cy-2-glu in vivo could influence their biodistribution pathways differently and be dependent on chemical factors of hydrophobicity, charge, and available functional groups for interaction.

Understanding the transport pathway of cypate contributes new knowledge to help optimize the chemical structure of such dyes. To validate our assumption that cypate uses carboxylates for transport purposes in vitro and to be responsible for the differences in uptake between cancer and normal cell lines, we performed competitive uptake inhibition experiments using PA, the fatty acid known to use the fatty acid transport mechanism.

Transport of fatty acids such as oleate, stearate, and palmitate is facilitated by membrane transport proteins (Abumrad et al., 1981, 1984; Potter et al., 1987). Long-chain fatty acids are mainly transported through CD36, plasma membrane-associated fatty acid-binding protein, and fatty acid transport proteins (Ehehalt et al., 2006; Schwenk et al., 2008; Su and Abumrad, 2009). The decrease in the fluorescence intensity of cypate in the presence of excess PA suggested that the binding sites for fatty acid were blocked by PA and were unavailable for cypate, confirming the use of carboxylates by cypate.

We synthesized a non-NIR fluorescent analog of cypate called sat-cy by adding four hydrogen atoms across the $\pi$-conjugated system in cypate, interrupting the $\pi$-conjugation system responsible for NIR optical properties. The presence of an excess of sat-cy also led to a decrease in the fluorescence intensity of cypate. Neither PA nor sat-cy exhibited competitive inhibition for the uptake of cy-2-glu, in which the carboxylic acid is modified to an amide bond. Thus, cypate and sat-cy may use the membrane transport proteins employed by fatty acids for entering cells.

Guo et al. (2012) found that both cypate and the glucosamine conjugates were cleared from the body within 24 hours, whereas Korotcov et al. (2012) showed that the retention time of the cypate-glucosamine conjugate was almost 96 hours. In our study we observed that cy-2-glu was retained in the animal for more than 6 days. This slow removal of cy-2-glu from the animal with increased tumor accumulation suggests that cy-2-glu could be a promising NIR probe for cancer detection and treatment monitoring.

The biodistribution data revealed that, second to the liver, the tumor had considerable accumulation of cy-2-glu, followed by the kidneys and spleen. Analysis of urine extracts by absorption spectroscopy and mass spectrometry revealed that this probe was cleared from the body through renal excretion in the form of free cypate.

It is possible that cy-2-glu undergoes hydrolysis in the liver, kidneys, and spleen which produces glucosamine and the cypate that is subsequently excreted and detected in urine. This possible hydrolysis was examined by performing an in vitro experiment with THLE-2 liver cells incubated with cy-2-glu; this experiment showed the presence of free cypate as one of the excreted metabolites. The mass spectrometry data of the in vitro extracts of prostate cancer cells as well as the normal cells revealed the presence of cypate, indicating deamidation of cy-2-glu. Fatty acid amide hydrolase and $N$-acylethanolamine acid amidase are the two main enzymes present in the animal tissue responsible for degradation of $N$-acylethanolamines into fatty acid and ethanolamine (Cravatt et al., 1996; Ueda et al., 1999, 2001; Tsuboi et al., 2005, 2007; Sakura et al., 2016). Cy-2-glu likely undergoes deamidation by these enzymes and releases free glucosamine.

In summary, the presence of excess D-glucose was observed to competitively inhibit the amount of cy-2-glu taken up in cancer cells both in vitro and in vivo, indicating cy-2-glu transport likely occurs through a glucose-mediated pathway and that the attachment of a bulky molecule like cypate does not alter the transport pathway of glucosamine. The presence of excess sat-cy and PA competitively decreased the amount of cypate transported into the cells, indicating the probable use of carboxylate groups for transport via the fatty acid transport proteins. The long retention period and high accumulation of cy-2-glu in tumors along with the release of glucosamine through hydrolysis in the tissues suggest that cy-2-glu has promising applications for preclinical animal imaging studies, especially with orthotopic and subcutaneous tumors. With further optimization cy-2-glu could have future translation use. 


\section{Acknowledgments}

We would like to acknowledge Dr. Griffith Parks, The Interim Associate Dean for research, for providing us the frozen vials of A549, BPH, DU145 and MDA-MB-231 cell lines, Kunal Dhume, BSBS, UCF, for helping in statistical analysis, Alexander Bosak, BSBS, Internal Medicine, UCF, for helping in grammar editing, and Dr. Chandrakala Aluganti Narasimhulu, BSBS, College of Medicine, UCF.

\section{Authorship Contributions}

Participated in research design: Doshi, Nierenberg, FloresFernandez, Khaled, Parthasarathy.

Conducted experiments: Doshi, Nierenberg, Flores-Fernandez, Deme.

Performed data analysis: Doshi, Nierenberg, Deme, Becerra.

Wrote of contributed to the writing of the manuscript: Doshi, Nierenberg, Deme, Khaled, Parthasarathy.

\section{References}

Abumrad NA, Park JH, and Park CR (1984) Permeation of long-chain fatty acid into adipocytes. Kinetics, specificity, and evidence for involvement of a membrane protein. $J$ Biol Chem 259:8945-8953.

Abumrad NA, Perkins RC, Park JH, and Park CR (1981) Mechanism of long chain fatty acid permeation in the isolated adipocyte. J Biol Chem 256:9183-9191.

Achilefu S, Bloch S, Markiewicz MA, Zhong T, Ye Y, Dorshow RB, Chance B, and Liang K (2005) Synergistic effects of light-emitting probes and peptides for targeting and monitoring integrin expression. Proc Natl Acad Sci USA 102:7976-7981.

Achilefu S, Dorshow RB, Bugaj JE, and Rajagopalan R (2000) Novel receptortargeted fluorescent contrast agents for in vivo tumor imaging. Invest Radiol 35:479-485.

Calvo MB, Figueroa A, Pulido EG, Campelo RG, and Aparicio LA (2010) Potential role of sugar transporters in cancer and their relationship with anticancer therapy. Int $J$ Endocrinol 2010:14.

Chan K, McMahon M, Liu G, Kato Y, Bhujwalla Z, Artemov D, and van Zijl P (2011) Imaging of glucose uptake in breast tumors using non-labeled D-glucose, in Proceedings of the International Society of Magnetic Resonance in Medicine, 19th Meeting and Exhibition; 2011 May 7-13; Montreal, Quebec, Canada. p. 551, International Society for Magnetic Resonance in Medicine (ISMRM), Concord, CA.

Cheng Z, Levi J, Xiong Z, Gheysens O, Keren S, Chen X, and Gambhir SS (2006) Near-infrared fluorescent deoxyglucose analogue for tumor optical imaging in cell culture and living mice. Bioconjug Chem 17:662-669.

Cook GMW and Jacobson W (1968) The electrophoretic mobility of normal and leukaemic cells of mice. Biochem $J$ 107:549-557.

Cravatt BF, Giang DK, Mayfield SP, Boger DL, Lerner RA, and Gilula NB (1996) Molecular characterization of an enzyme that degrades neuromodulatory fatty-acid amides. Nature 384:83-87.

Donuru VR, Zhu S, Green S, and Liu H (2010) Near-infrared emissive bodipy polymeric and copolymeric dyes. Polymer (Guildf) 51:5359-5368.

Ebstensen RD and Plagemann PGW (1972) Cytochalasin B: inhibition of glucose and glucosamine transport. Proc Natl Acad Sci USA 69:1430-1434.

Effert P, Beniers AJ, Tamimi Y, Handt S, and Jakse G (2004) Expression of glucose transporter 1 (Glut-1) in cell lines and clinical specimens from human prostate adenocarcinoma. Anticancer Res 24 (5A):3057-3063.

Ehehalt R, Füllekrug J, Pohl J, Ring A, Herrmann T, and Stremmel W (2006) Translocation of long chain fatty acids across the plasma membrane--lipid rafts and fatty acid transport proteins. Mol Cell Biochem 284:135-140.

Escobedo JO, Rusin O, Lim S, and Strongin RM (2010) NIR dyes for bioimaging applications. Curr Opin Chem Biol 14:64-70.

Flamen P, Lerut A, Van Cutsem E, De Wever W, Peeters M, Stroobants S, Dupont P, Bormans G, Hiele M, De Leyn P, et al. (2000) Utility of positron emission tomography for the staging of patients with potentially operable esophageal carcinoma. $J$ Clin Oncol 18:3202-3210.

Guo J, Du C, Shan L, Zhu H, Xue B, Qian Z, Achilefu S, and Gu Y (2012) Comparison of near-infrared fluorescent deoxyglucose probes with different dyes for tumor diagnosis in vivo. Contrast Media Mol Imaging 7:289-301.

Hanahan D and Weinberg RA (2011) Hallmarks of cancer: the next generation. Cell 144:646-674

Haque A, Faizi MSH, Rather JA, and Khan MS (2017) Next generation NIR fluorophores for tumor imaging and fluorescence-guided surgery: a review. Bioorg Med Chem 25:2017-2034.

Hilderbrand SA and Weissleder R (2010) Near-infrared fluorescence: application to in vivo molecular imaging. Curr Opin Chem Biol 14:71-79.

Hoh CK, Hawkins RA, Glaspy JA, Dahlbom M, Tse NY, Hoffman EJ, Schiepers C, Choi Y, Rege S, Nitzsche E, et al. (1993) Cancer detection with whole-body PET using 2-[18F]fluoro-2-deoxy-D-glucose. J Comput Assist Tomogr 17:582-589.

James AM, Ambrose EJ, and Lowick JHB (1956) Differences between the electrical charge carried by normal and homologous tumour cells. Nature 177:576-577.

Kim JS, Kodagahally R, Strekowski L, and Patonay G (2005) A study of intramolecular H-complexes of novel bis(heptamethine cyanine) dyes. Talanta 67:947-954.

Korotcov AV, Ye Y, Chen Y, Zhang F, Huang S, Lin S, Sridhar R, Achilefu S, and Wang PC (2012) Glucosamine-linked near-infrared fluorescent probes for imaging of solid tumor xenografts. Mol Imaging Biol 14:443-451.
Lapela M, Grénman R, Kurki T, Joensuu H, Leskinen S, Lindholm P, Haaparanta M, Ruotsalainen U, and Minn H (1995) Head and neck cancer: detection of recurrence with PET and 2-[F-18]fluoro-2-deoxy-D-glucose. Radiology 197: 205-211.

Liberti MV and Locasale JW (2016) The Warburg effect: how does it benefit cancer cells? Trends Biochem Sci 41:211-218.

Luo S, Zhang E, Su Y, Cheng T, and Shi C (2011) A review of NIR dyes in cancer targeting and imaging. Biomaterials 32:7127-7138.

Medina RA and Owen GI (2002) Glucose transporters: expression, regulation and cancer. Biol Res 35:9-26.

Nakazumi H, Ohta T, Etoh H, Uno T, Colyer CL, Hyodo Y, and Yagi S (2005) Nearinfrared luminescent bis-squaraine dyes linked by a thiophene or pyrene spacer for noncovalent protein labeling. Synth Met 153:33-36.

O'Byrne KJ, Baird A-M, Kilmartin L, Leonard J, Sacevich C, and Gray SG (2011) Epigenetic regulation of glucose transporters in non-small cell lung cancer. Cancers (Basel) 3:1550-1565.

Ong LC, Jin Y, Song IC, Yu S, Zhang K, and Chow PK (2008) 2-[18F]-2-deoxy-Dglucose (FDG) uptake in human tumor cells is related to the expression of GLUT-1 and hexokinase II. Acta Radiol 49:1145-1153.

Owens EA, Henary M, El Fakhri G, and Choi HS (2016) Tissue-specific near-infrared fluorescence imaging. Acc Chem Res 49:1731-1740

Peng X, Song F, Lu E, Wang Y, Zhou W, Fan J, and Gao Y (2005) Heptamethine cyanine dyes with a large Stokes shift and strong fluorescence: a paradigm for excited-state intramolecular charge transfer. $J$ Am Chem Soc 127: 4170-4171.

Phelps ME (2000) Positron emission tomography provides molecular imaging of biological processes. Proc Natl Acad Sci USA 97:9226-9233.

Plagemann PG and Erbe J (1973) Transport and metabolism of glucosamine by cultured Novikoff rat hepatoma cells and effects on nucleotide pools. Cancer Res 33: 482-492.

Potter BJ, Stump D, Schwieterman W, Sorrentino D, Jacobs LN, Kiang CL, Rand JH, and Berk PD (1987) Isolation and partial characterization of plasma membrane fatty acid binding proteins from myocardium and adipose tissue and their relationship to analogous proteins in liver and gut. Biochem Biophys Res Commun 148:1370-1376.

Purdom L, Ambrose EJ, and Klein G (1958) A correlation between electrical surface charge and some biological characteristics during the stepwise progression of a mouse sarcoma. Nature 181:1586-1587.

Rivlin M and Navon G (2018) CEST MRI of 3-O-methyl-D-glucose on different breast cancer models. Magn Reson Med 79:1061-1069.

Sakura Y, Tsuboi K, Uyama T, Zhang X, Taoka R, Sugimoto M, Kakehi Y, and Ueda N (2016) A quantitative study on splice variants of N-acylethanolamine acid amidase in human prostate cancer cells and other cells. Biochim Biophys Acta 1861 (12 Pt A):1951-1958.

Schwenk RW, Luiken JJ, Bonen A, and Glatz JF (2008) Regulation of sarcolemmal glucose and fatty acid transporters in cardiac disease. Cardiovasc Res 79: 249-258.

Som P, Atkins HL, Bandoypadhyay D, Fowler JS, MacGregor RR, Matsui K, Oster ZH, Sacker DF, Shiue CY, Turner H, et al. (1980) A fluorinated glucose analog, 2-fluoro-2-deoxy-D-glucose (F-18): nontoxic tracer for rapid tumor detection. $J$ Nucl Med 21:670-675.

Srinivasan A, Ishizuka T, Osuka A, and Furuta H (2003) Doubly N-confused hexaphyrin: a novel aromatic expanded porphyrin that complexes bis-metals in the core. J Am Chem Soc 125:878-879.

Su X and Abumrad NA (2009) Cellular fatty acid uptake: a pathway under construction. Trends Endocrinol Metab 20:72-77.

Tanaka Y, Shin J-Y, and Osuka A (2008) Facile synthesis of large meso-pentafluorophenylsubstituted expanded porphyrins. Eur J Org Chem 2008:1341-1349.

Tsuboi K, Sun Y-X, Okamoto Y, Araki N, Tonai T, and Ueda N (2005) Molecular characterization of $\mathrm{N}$-acylethanolamine-hydrolyzing acid amidase, a novel member of the choloylglycine hydrolase family with structural and functional similarity to acid ceramidase. J Biol Chem 280:11082-11092.

Tsuboi K, Takezaki N, and Ueda N (2007) The $N$-acylethanolamine-hydrolyzing acid amidase (NAAA). Chem Biodivers 4:1914-1925.

Ueda N, Yamanaka K, Terasawa Y, and Yamamoto S (1999) An acid amidase hydrolyzing anandamide as an endogenous ligand for cannabinoid receptors. FEBS Lett 454:267-270.

Ueda N, Yamanaka K, and Yamamoto S (2001) Purification and characterization of an acid amidase selective for $N$-palmitoylethanolamine, a putative endogenous anti-inflammatory substance. J Biol Chem 276:35552-35557.

Umezawa K, Citterio D, and Suzuki K (2008a) Water-soluble NIR fluorescent probes based on squaraine and their application for protein labeling. Anal Sci 24: $213-217$

Umezawa K, Matsui A, Nakamura Y, Citterio D, and Suzuki K (2009) Bright, color-tunable fluorescent dyes in the Vis/NIR region: establishment of new "tailor-made" multicolor fluorophores based on borondipyrromethene. Chemistry 15:1096-1106.

Umezawa K, Nakamura Y, Makino H, Citterio D, and Suzuki K (2008b) Bright, colortunable fluorescent dyes in the visible-near-infrared region. J Am Chem Soc 130:1550-1551.

Vinogradov E, Sherry AD, and Lenkinski RE (2013) CEST: from basic principles to applications, challenges and opportunities. J Magn Reson 229:155-172.

Volkova KD, Kovalska VB, Tatarets AL, Patsenker LD, Kryvorotenko DV, and Yarmoluk SM (2007) Spectroscopic study of squaraines as protein-sensitive fluorescent dyes. Dyes Pigments 72:285-292.

Walker-Samuel S, Ramasawmy R, Torrealdea F, Rega M, Rajkumar V, Johnson SP, Richardson S, Goncalves M, Parkes HG, Årstad E, et al. (2013) In vivo imaging of glucose uptake and metabolism in tumors. Nat Med 19:1067-1072.

Warburg O (1925) The metabolism of carcinoma cells. J Cancer Res 9:148-163.

Warburg O (1956) On the origin of cancer cells. Science 123:309-314. 
Wu B, Warnock G, Zaiss M, Lin C, Chen M, Zhou Z, Mu L, Nanz D, Tuura R, and Delso G (2016) An overview of CEST MRI for non-MR physicists. EJNMMI Phys 3:19.

Xie Y-S, Yamaguchi K, Toganoh M, Uno H, Suzuki M, Mori S, Saito S, Osuka A, and Furuta H (2009) Triply N-confused hexaphyrins: near-infrared luminescent dyes with a triangular shape. Angew Chem Int Ed Engl 48:5496-5499.

Ye Y, Bloch S, Kao J, and Achilefu S (2005) Multivalent carbocyanine molecular probes: synthesis and applications. Bioconjug Chem 16:51-61.

Zhang S, Trokowski R, and Sherry AD (2003) A paramagnetic CEST agent for imaging glucose by MRI. J Am Chem Soc 125:15288-15289.
Zhang Y, Yang M, Portney NG, Cui D, Budak G, Ozbay E, Ozkan M, and Ozkan CS (2008) Zeta potential: a surface electrical characteristic to probe the interaction of nanoparticles with normal and cancer human breast epithelial cells. Biomed Microdevices 10:321-328.

Address correspondence to: Dr. Sampath Parthasarathy, Burnett School of Biomedical Sciences, College of Medicine, University of Central Florida, 6900 Lake Nona Blvd, Orlando, FL 32827. E-mail: spartha@ucf.edu 